\title{
Novel systems analysis elucidates the influence of personalized variation in microbiome composition on metronidazole efficacy in bacterial vaginosis
}

\section{Christina Lee}

University of Michigan

\section{Ryan Cheu}

University of Miami

Melissa Lemke

University of Michigan-Ann Arbor https://orcid.org/0000-0003-1860-4550

Andrew Gustin

University of Washington https://orcid.org/0000-0002-8760-8320

Michael France

University of Maryland, Baltimore

\section{Benjamin Hampel}

University of Zurich

\section{Andrea Thurman}

Eastern Virginia Medical School

\section{Gustavo Doncel}

Eastern Virginia Medical School

Jacques Ravel

University of Maryland, Baltimore https://orcid.org/0000-0002-0851-2233

Nichole Klatt

University of Miami

Kelly Arnold ( $\sim$ kbarnold@umich.edu )

University of Michigan-Ann Arbor

\section{Article}

Keywords: systems analysis, bacterial vaginosis (BV), metronidazole efficacy

Posted Date: September 3rd, 2020

DOI: https://doi.org/10.21203/rs.3.rs-66397/v1 
License: (c) (i) This work is licensed under a Creative Commons Attribution 4.0 International License. Read Full License

Version of Record: A version of this preprint was published at Nature Communications on December 1st, 2020. See the published version at https://doi.org/10.1038/s41467-020-19880-w. 
Novel systems analysis elucidates the influence of personalized variation in microbiome composition on metronidazole efficacy in bacterial vaginosis

Christina Y. Lee ${ }^{1 *}$, Ryan K. Cheu ${ }^{2,3^{*}}$, Melissa M. Lemke ${ }^{1}$, Andrew Gustin ${ }^{2,3}$, Michael France ${ }^{4}$, Benjamin Hampel ${ }^{5}$, Andrea Thurman 6 , Gustavo F. Doncel ${ }^{6}$, Jacques Ravel ${ }^{4}$, Nichole R. Klatt ${ }^{2,3 \#}$, Kelly B. Arnold ${ }^{1 \#}$

${ }^{1}$ Department of Biomedical Engineering, University of Michigan, Ann Arbor, MI, United States of America

${ }^{2}$ University of Miami Department of Pediatrics, University of Miami, Miami, FL, United States of America

${ }^{3}$ Department of Pharmaceutics, University of Washington, Seattle, WA, United States of America

${ }^{4}$ Institute for Genome Sciences and Department of Microbiology and Immunology, University of Maryland School of Medicine, Baltimore, MD, 21201, United States of America

${ }^{5}$ Division of Infectious Diseases and Hospital Epidemiology, University of Zurich, 8901, Zürich, Switzerland

${ }^{6}$ Eastern Virginia Medical School, CONRAD, Norfolk, VA, United States of America 
*These authors contributed equally to this work.

${ }^{\#}$ co-senior authors

Address correspondence to: Kelly B. Arnold (kbarnold@umich.edu), Nicole R. Klatt ( nklatt@med.miami.edu) 


\section{Abstract}

2 Bacterial vaginosis (BV) is a syndrome of the female reproductive tract associated with

3 adverse reproductive outcomes and characterized by a shift from a Lactobacillus (LB)-

4 dominant vaginal microbiota to a polymicrobial, anaerobic microbiota, consistently

5 colonized by strains of Gardnerella vaginalis $(\mathbf{G v})$. The first-line treatment for BV is

6 metronidazole (MNZ); however, treatment failure and recurrence rates remain high. To

7 gain insight into complex interactions between target species (Gv) and non-target

8 Lactobacillus species (Lactobacillus iners (Li)) with MNZ and understand their respective

9 roles in efficacy, we developed an ordinary differential equation model that predicts

10 bacterial growth as a function of drug uptake, metabolism, proliferation, and MNZ

11 sensitivity. Model findings revealed a critical factor in MNZ efficacy may be $L i$

12 sequestration of MNZ, and that efficacy decreases when the relative abundance of $\mathrm{Li}$ is

13 higher pre-treatment. These results were validated in $G v$ and $L i$ co-cultures $(p<0.001)$,

14 and in two clinical cohorts, finding women with recurrent BV had significantly lower pre-

15 treatment levels of BV-associated bacteria relative to Lactobacillus spp. $(p=0.0366 ; p=$

16 0.0484). Overall, model results support a mechanism where non-target Lactobacillus

17 species sequester MNZ from BV-associated target species, such as Gv, promoting BV

18 recurrence by reducing MNZ bioavailability. 
Bacterial vaginosis (BV) is a condition that affects $30-60 \%$ of women worldwide,,$^{1,2}$

21 with negative outcomes involving increased susceptibility to sexually transmitted

22 infections (STIs), and adverse reproductive outcomes including pre-term birth. ${ }^{3-7}$ BV is

23 characterized by a shift from Lactobacillus species (spp.)-dominated vaginal microbiota

24 to a wide array of anaerobic bacteria including Gardnerella vaginalis ( $G v)$ and Atopobium

25 vaginae. ${ }^{8,9,10}$ Treatment of symptomatic BV with metronidazole (MNZ) is aimed to restore

26 Lactobacillus-dominated microbiota, but because BV is not caused by a single pathogen

27 easily targeted with antimicrobial therapy, recurrence rates are reported to be $57-90 \%$

28 within 12 months of receiving adequate treatment. ${ }^{11,12,13,14}$ Recurrence is associated with

29 several host factors including previous episodes of BV, douching, and sexual activity, but

30 no one factor emerges as a single driver of treatment failure. ${ }^{11,15-17,18}$ Additionally,

31 associations between vaginal microbiota composition and BV recurrence have been

32 reported but remain poorly understood, with several studies citing conflicting

33 results. ${ }^{15,17,19}$

Recent improvements in 16S rRNA sequencing have enhanced the ability to

35 identify and more accurately quantify the composition of the vaginal microbiota in $\mathrm{BV}, 20,21$

36 finding that the transition is frequently associated with an abundance of Lactobacillus

37 iners $(\boldsymbol{L} i){ }^{22,23}$ Despite the association between $L i$ and incidence of $\mathrm{BV}$, identifying how $L i$

38 dictates communities of optimal and non-optimal microbiota remains elusive, as the

39 vaginal microbiota can change significantly over time and vary between women $24,25,26$,

40 especially in the presence of MNZ. The recommended treatment regimen for BV consists

41 of oral or vaginal MNZ oriented towards selectively targeting anaerobic bacteria with little 
42 effect on Lactobacillus spp., ${ }^{27,28}$ but high variability in efficacy indicates that further study

43 is required to understand the reestablishment of optimal vaginal microbiota ecosystems.

$44 \quad$ Recent research in the HIV microbicide field has highlighted the importance of 45 vaginal microbiome composition in drug treatment efficacy. In a landmark study, variability 46 in tenofovir (TFV) microbicide efficacy was accounted for by differences in the vaginal

47 microbiome, specifically the presence of the non-target species Gv, which were shown to 48 metabolize TFV. ${ }^{29}$ Likewise for MNZ treatment of Trichomonas vaginalis, a proposed 49 mechanism of treatment failure was decreased bioavailability of MNZ due to the 50 absorbance of the antibiotic by other microorganisms in the vagina. ${ }^{30,31,32}$ In the context 51 of $\mathrm{BV}$, it is possible that the mechanisms underlying MNZ treatment failure could be 52 related to complex inter-species growth dynamics and absorption of MNZ. We propose 53 that variability in MNZ efficacy may result from underlying differences in MNZ uptake and 54 susceptibility in target and non-target species, and therefore would be highly dependent 55 on individual differences in pre-treatment vaginal microbiota composition.

56 To test this hypothesis, we developed an ordinary differential equation (ODE)

57 model that uses experimentally measured parameters (MNZ internalization by bacteria,

58 metabolism and bacterial antibiotic susceptibility) to predict $L i$ and Gv growth dynamics 59 with MNZ treatment. Ultimately, this model revealed the surprising insight that a critical 60 factor in MNZ efficacy may be Li sequestration of MNZ, and that efficacy is predicted to 61 be significantly reduced in individuals with higher pre-treatment amounts of the non-target 62 species $L i$ relative to the target species $G v$. We experimentally validated this finding with 63 in vitro co-cultures, and used more complex computational frameworks to illustrate that 64 this behavior would also be expected in microbial environments with additional species, 
65 interspecies interactions and stain variability. Finally, we validated results in

66 cervicovaginal samples from BV-infected women treated with MNZ in two different clinical

67 cohorts. ${ }^{33,34}$ We propose that quantitative evaluation of target bacteria and non-target

68 Lactobacillus spp. interactions with MNZ will provide new insight into personalized

69 differences in BV recurrence and treatment failure.

71 Results

72 Computational ODE model elucidates an important role for $L$. iners sequestration of MNZ

73 from $G$. vaginalis

To determine how MNZ treatment efficacy can be altered by bacterial-mediated

75 interactions in vitro, we created an ODE model to predict growth of Gv and Li upon co-

76 culture and treatment with MNZ (Fig. 1a). Parameters for each bacterial species were

77 obtained by least squares fitting of in vitro kinetic data and dose-response curves for MNZ

78 exposure with each species in monoculture (fig. S1, S2, Table S1), before the ODE model

79 was used to predict co-culture conditions with Gv and Li both interacting with extracellular

80 MNZ. The model assumes that $G v$ and $L i$ internalize or sequester MNZ at rates kint-Gv and

81 kint-Li, respectively, and Gv can convert MNZ to the stable metabolite, acetamide and

82 unknown metabolites at rate $\mathrm{kmet}^{35}$ The model additionally assumes logistic growth at

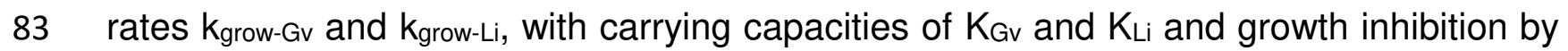

84 MNZ toxicity at rates $\mathrm{k}_{\text {kill-Gv }}$ and $\mathrm{k}_{\text {kill-Li }}$ in a dose-dependent manner based on $50 \%$ effective

85 concentrations of MNZ on Gv and $\mathrm{Li}(\mathrm{EC} 50 \mathrm{Gv}, \mathrm{EC} 50 \mathrm{Li}) \cdot{ }^{36,37}$ Since MNZ is a pro-drug that

86 is activated when internalized by anaerobic bacteria, the cytotoxicity of MNZ in the model 
87 is dependent on the intracellular concentration of MNZ rather than extracellular MNZ

88 concentration; however, we used the external MNZ concentration as the basis for EC50

89 of internalized MNZ, as experimentally determining the intracellular level of MNZ per cell

90 was challenging and the main goal was to capture the relative sensitivity between Gv and

$91 \quad L i^{27,28,30,38}$

92 To identify model parameters that were most critical for decreasing Gv growth, we

93 performed a 1-dimensional (1D) sensitivity analysis by altering each parameter three

94 orders of magnitude above and below baseline and evaluated Gv growth (Fig. 1b \& c).

95 Growth was scaled relative to the predicted growth in an unperturbed co-culture based

96 on the time point and initial population sizes evaluated and is referred to as percent

97 maximum growth. The sensitivity analysis identified Gv growth as highly dependent on

98 the MNZ internalization/sequestration rate into $L i$ ( $k_{\text {int-Li). }}$. A 50 -fold increase in this rate

99 increased the growth of Gv from $7.42 \%$ to $69.5 \%$ its maximal growth upon $48 \mathrm{~h}$ treatment

100 with MNZ, where percent maximal growth describes the expected proportion of cell

101 density with antibiotic treatment relative to cell density with the same initial culture

102 conditions without antibiotic (Fig. 1c). Likewise, changing the MNZ internalization rate

103 into Gv (kint-Gv) has similar effects on $L i$, where increasing this rate 50 -fold resulted in

$10489.7 \%$ Li's maximal growth (fig. S3a). Overall, these results illustrate how MNZ efficacy

105 in inhibiting Gv growth is influenced by the competition between each bacterium to 106 internalize the drug.

From this result we hypothesized that the relative quantity of cells internalizing

108 MNZ (ratio of $G v$ and $L I$ ) could significantly influence growth of both strains. We tested 109 this hypothesis in our computational framework by predicting Gv survival after varying the 
110 starting ratio of $G v$ to $L i$ (Gv:Li ratio) from 1,000x fold to $0.001 x$. Results indicated that

111 altering the initial Gv:Li ratio influences the growth of both Gv and Li. Counterintuitively,

$112 G v$ survival was high when $L i$ initially outnumbers Gv 1,000x and $L i$ growth is optimal

113 when Gv initially exceeds $L i$ 1,000x (Fig. 1d). Stated differently, the model suggested

114 that more Gv present at MNZ treatment initiation resulted in a better treatment outcome.

115 The importance of MNZ internalization rate into $L i$ became more apparent as $L i$ became

116 the predominating species, leading to increased growth of Gv (Fig. 1e). This result

117 additionally supports that $L i$ competes with $G v$ to internalize or sequester extracellular

118 MNZ, as when one bacterial strain is in excess, it likely depletes available extracellular

119 MNZ and decreases the amount of drug internalized by the non-dominating bacterial 120 strain.

121 We used our model to explore this ratio-dependent behavior over a range of

122 relevant MNZ concentrations extending from $100 \mu \mathrm{g} / \mathrm{ml}$ to $1,600 \mu \mathrm{g} / \mathrm{ml}$, as estimates for

123 vaginal accumulation range from $20 \mu \mathrm{g} / \mathrm{ml}$ to greater than $1,000 \mu \mathrm{g} / \mathrm{ml}$ (Fig. 2a, b). ${ }^{39,40}$

124 Doses below $100 \mu \mathrm{g} / \mathrm{ml}$ had no effect on Gv or Li growth and doses above 1,600 $\mu \mathrm{g} / \mathrm{ml}$

125 exhibited near complete cell killing for both bacterial strains (Fig. 2a \& b); these data are

126 in agreement with experimentally determined effective concentrations of MNZ on Gv and

$127 L i$ cultured individually (fig. S2 c-d). However, for doses between 100 and 1,600 $\mu \mathrm{g} / \mathrm{ml}$

128 there were significant differences dependent on the initial Gv:Li ratio, where MNZ was

129 most efficacious in eliminating Gv when more Gv than Li was present. 
We validated these counterintuitive model predictions experimentally in vitro by

134 varying the initial Gv:Li ratios in the presence of $500 \mu \mathrm{g} / \mathrm{ml} \mathrm{MNZ} \mathrm{and} \mathrm{tracking} \mathrm{growth} \mathrm{for}$

135 48h (Fig. 2c, d). Experimental measurements confirmed model predictions that MNZ

136 efficacy for inhibiting Gv growth decreased when Li was initially dominant $(p<0.0001, t$

$137=6.985, \mathrm{df}=34)$, and were not significantly different than model predictions $(0.001 \times \mathrm{Gv}: \mathrm{Li}$,

$138 p=0.4297, t=0.8087, d f=17 ; 1,000 x$ Gv:Li ratio, $p=0.6891, t=0.4070, d f=17 F i g .3 c)$,

139 with Gv exhibiting a predicted $30.3 \%$ and experimental $41.4 \% \pm 13.3 \%$ maximal growth

140 after treatment when $\mathrm{Li}$ was initially dominant compared to a predicted $2.1 \%$ and

141 experimental $9.4 \% \pm 13.8 \%$ maximal growth when Gv initially was dominant. Li growth in

142 the presence of $500 \mu \mathrm{g} / \mathrm{ml}$ MNZ was also dependent on the initial Gv:Li ratio, where MNZ

143 inhibited Li growth the most when Li was initially dominant, $7.2 \% \pm 3.9 \%$ maximal growth

144 compared to when Gv was initially dominant, $70.5 \% \pm 33.8 \%(p<0.0001, t=7.908, d f=$

145 34, Fig. 2d). Notably, the model over-predicted the growth of the Li population when $\mathrm{Li}$

146 was initially dominant $(0.001 \times \mathrm{Gv}: \mathrm{Li})$, where the model prediction of $23.9 \%$ maximal

147 growth was over 3 -fold higher than experimentally observed, $7.19 \% \pm 3.91 \%$ growth

148 (0.001x Gv:Li experiment vs simulation, $\mathrm{p}<0.0001, \mathrm{t}=18.15, \mathrm{df}=17)$, suggesting 149 efficacy dependence on a high pre-treatment Gv:Li ratio may be even greater than that

150 predicted by the model. Experimental and model predictions of Li growth were not

151 significantly different when Gv was initially dominant $(1,000 \times \mathrm{GV}: \mathrm{LI}, \mathrm{p}=0.1970, \mathrm{t}=1.343$,

$152 \mathrm{df}=17)$. Likewise, model predictions of MNZ and MNZ metabolite concentrations were

153 not significantly different from experimental results in cultures starting with a 0.001x Gv:Li

154 ratio (extracellular $\mathrm{MNZ}: \mathrm{p}=0.2545, \mathrm{t}=1.178, \mathrm{df}=17$, intracellular $\mathrm{MNZ}: \mathrm{p}=0.3356, \mathrm{t}=$ 
$1550.9910, \mathrm{df}=17$ acetamide: $\mathrm{p}=0.8341, \mathrm{t}=0.1567, \mathrm{df}=17$ ), but predictions for extracellular

156 MNZ, intracellular MNZ, and acetamide concentrations in cultures with a 1,000x Gv:Li

157 ratio did vary significantly from experimental data (fig. S4). The deviation of model

158 predictions when $G v$ is initially dominant suggests that experimental investigation of

159 detailed mechanisms of Gv interactions with MNZ is warranted (for example the potential

160 ability of $G v$ to externally degrade MNZ). Despite some deviation of peripheral model

161 predictions from experimental measurements, the Gv:Li ratio-dependent trends were

162 reproduced by the model. The dependency on initial culture ratios of Gv to Li on growth

163 suggests that non-target bacteria that sequester MNZ could significantly alter drug

164 efficacy.

165

We observed some variation in the sensitivity (EC50) of $L i$ to MNZ. Variability in

166 minimum inhibitory concentrations (MIC) estimations have been reported, as changes in

167 culture conditions including incubation length and the inoculum effect can influence the

168 apparent sensitivity of bacteria to antibiotic. ${ }^{37,41}$ Additionally, the sensitivity of

169 Lactobacillus sp. and Gv to MNZ and their MICs are reported to range from $500 \mu \mathrm{g} / \mathrm{ml}-$

$1704,000 \mu \mathrm{g} / \mathrm{ml}$ and $0.75 \mu \mathrm{g} / \mathrm{ml}$ to greater than $256 \mu \mathrm{g} / \mathrm{ml}$, respectively. ${ }^{42-44}$ To ascertain

171 whether our results would be influenced by variation in Li sensitivity to MNZ, we repeated

172 the simulations over a range of EC50 values. To represent reported resistance of

173 Lactobacillus sp. in vitro, we increased the EC50 value of $L i$ to be 10 -fold higher than Gv

$174(E C 50\llcorner i=4,200 \mu \mathrm{g} / \mathrm{ml})$. MNZ efficacy in inhibiting Gv growth was similarly decreased at

175 low Gv:Li ratios (36.5\% max growth at $0.001 \times$ Gv:Li) compared to high Gv:Li ratios $(3.96 \%$

176 max growth at $1000 x \mathrm{Gv}$ :Li, fig. S5a). Li had little to no susceptibility over the range of

177 MNZ concentrations tested (fig. S5b). Additionally, these EC50 values replicated trends 
178 in experimental data for growth kinetics (fig. S2d \& h versus S5c \& d). These results

179 support that the initial Gv:Li ratio dependent trends in MNZ efficacy for inhibiting Gv 180 growth are independent of Li's sensitivity to MNZ.

181 The model identifies optimal MNZ doses that can shift a $G$. vaginalis dominant population

182 to a L. iners dominant population in-vitro

We next used the model to determine specific combinations of MNZ concentrations

184 and initial Gv:Li ratios that resulted in optimal final Li proportion after 48 MNZ exposure.

185 The initial Gv:Li ratio was highly associated with the final Gv:Li ratio for doses of MNZ

186 greater than $250 \mu \mathrm{g} / \mathrm{ml}$ (Fig. 3a). Interestingly, cultures that were initially Li dominant

187 (0.001x Gv:LI), were nearly insensitive to any dose of MNZ, resulting consistently with

$188>50 \%$ Gv (Fig. 3a). This result carries the surprising implication that women with $\mathrm{Li}$ -

189 dominant vaginal microbiomes at treatment initiation are likely to undergo recurrence,

190 regardless of MNZ dose. Of note, cultures that were originally Gv dominant (Gv:Li > 1)

191 were the most likely to be $L i$ dominated after $48 \mathrm{~h}$ exposure to MNZ. Experimental data 192 supported these trends, as the simulation predictions were not significantly different for 193 the final proportion of $L i$ at $500 \mu \mathrm{g} / \mathrm{ml}$ for $1,000 \times(\mathrm{p}=0.6798, \mathrm{t}=0.4202$, $\mathrm{df}=17)$. The 194 model did over-estimate the final proportion of $L i$ at the $0.001 \times$ Gv:Li ratio (predicting a $19544.1 \%$ proportion of $L i$ compared to $14.2 \% \pm 7.16 \%$ obtained experimentally); however, 196 this result suggests an even more significant reduction in Liproportion when Gv is initially 197 dominant $(p=0.008, t=4.056, d f=17)$.

A phase diagram of MNZ therapy outcomes at $48 \mathrm{~h}$ was created to characterize 199 both $L i$ and Gv endpoint growth dynamics, which depict either an increase/expansion or 200 decrease in population size relative to the initial population. The optimal growth dynamics 
201 would depict the expansion of only the Li population and the least optimal growth

202 dynamics would be the expansion of only Gv. A decrease in both populations is

203 additionally not optimal, as lower levels of beneficial microbiota are often associated with

204 opportunistic infections or overgrowth of non-optimal species. ${ }^{45,46}$ We observed that

205 higher initial Gv:Li ratios in conjunction with MNZ concentrations over $250 \mu \mathrm{g} / \mathrm{mL}$ were

206 more likely to result in optimal final growth dynamics where the $L i$ bacterial population

207 was the only population expanding (Fig. 3b). Likewise, it was possible for only the Gv

208 population to grow and the Li population to decrease when the initial Gv:Li ratio was less

209 than $1 \mathrm{x}$. Interestingly, the diagram predicts that it is possible that both $\mathrm{Gv}$ and $\mathrm{Li}$

210 populations would decrease for intermediate ratios of Gv:Li, which expand to include a

211 wider range of ratios as the dose of MNZ is increased. Overall, in vitro co-culture

212 experimental data supported the model predictions for endpoint growth dynamics, with

21315 of 18 samples agreeing with the dynamics predicted by the phase diagram for the

$2141,000 \times$ Gv:Li, $500 \mu \mathrm{g} / \mathrm{ml}$ group and for all 18 samples agreeing with the predictions for

215 the $0.001 \times$ Gv:Li ratio, $500 \mu \mathrm{g} / \mathrm{ml}$ group (Fig. 3b, right). This result reinforces the

216 importance of pre-treatment Gv:Li ratio on post-treatment bacterial community

217 composition.

219 The pre-treatment Gv:Li ratio and MNZ sequestration by Li is predicted to influence MNZ 220 efficacy in microbial environments with interspecies interactions and strain variability

221 While our model results emphasize the importance of pre-treatment Gv:Li ratios in

222 MNZ efficacy in co-cultures, BV in women is more complex, and involves interspecies

223 interactions and strain variability across many different bacterial species. In order to 
224 evaluate the above results in more complex settings that include multiple species,

225 interspecies interactions, and strain variability, we created three additional model

226 structures (Fig. 4a-d). In Model B and Model D, we account for potential interspecies

227 interactions, such as amensalism between Lactobacillus spp. and BV-associated bacteria

228 and commensal or mutualistic behavior within BV-associated bacteria subpopulations

229 and Lactobacillus spp. (Fig. 4a \& d). ${ }^{47-51}$ In Models C and D we add additional

230 representative species; a second BV-associated species and second Lactobacillus sp.

231 (Fig. 4c \& d). In order to address potential variability in associated parameters, we

232 randomly selected parameter values from physiologically relevant ranges determined

233 from previously published studies (Table S2 \& S3). Notably, across all four model

234 structures we found that higher initial relative amounts of BV-associated bacteria to

235 Lactobacillus spp. had higher relative post-antibiotic levels of Lactobacillus spp. (BV:LB

236 ratio, Fig. 4e-f, fig. S6, $P<1 E-6, P<1 E-6, P<1 E-6, P<1 E-6)$. This result held for a

237 range of ratios $(0.6 \times \mathrm{BV}: \mathrm{LB}$ and $100 \times \mathrm{BV}: \mathrm{LB})$ chosen to reflect the observed relative

238 abundance of Lactobacillus spp. in BV positive women $(60 \%-1.0 \%){ }^{52}$ Moreover, for

239 each of these model structures, the global sensitivity analyses consistently selected the

240 MNZ internalization/sequestration parameter $\left(k_{\text {int }}\right)$ and the initial relative abundance of

241 BV-associated bacteria to Lactobacillus sp. (BV:LB ratio) as significantly sensitive

242 parameters in post-antibiotic treatment Lactobacillus spp. relative abundance. Variability

243 in Gv sensitivity to MNZ (EC50) and growth rate were also selected as critical parameters

244 in dictating response to MNZ treatment, which of interest as there is significant variability

245 across Gv subclasses in terms of resistance to MNZ, and metabolism. ${ }^{53}$ Furthermore,

246 when models were modified such that Lactobacillus spp. could not internalize/sequester 
247 MNZ, the ratio-dependent effect was abrogated, and was additionally independent of

248 sensitivity of Lactobacillus spp. to MNZ (fig. S7a-b). Altogether, this provides additional

249 quantitative evidence that Lactobacillus spp. sequestration of MNZ may contribute to BV

250 recurrence in more complex microbial environments.

252 Decreased pre-treatment $B V: L B$ ratio is associated with $B V$ recurrence in clinical samples

253 We next evaluated whether the influence of initial BV:LB ratio on MNZ efficacy is

254 observed clinically. We compared the pre-treatment ratio of BV-associated bacteria to

255 Lactobacillus spp. (BV:LB ratio) in vaginal samples collected from women who underwent

256 MNZ treatment for BV and were cured or experienced recurrence, in two clinical studies;

257 the UMB-HMP study ${ }^{33}(n=11)$ and CONRAD BV study ${ }^{34}(n=33)$. We chose to evaluate

258 each study separately to minimize effects of differences in sample collection and in

259 methods of microbial species measurements. In the UMB-HMP cohort, 11 women were

260 observed over the course of 10 weeks and provided cervicovaginal lavage (CVLs)

261 samples each day for quantification of relative microbial abundances by sequencing of

262 the V3 and V4 regions of $16 \mathrm{~S}$ rRNA. Patients underwent treatment for BV that consisted

263 of one week of 500-mg oral MNZ, taken twice daily. Of the 11 women, 8 met inclusion

264 criteria and were classified as recurrent or cured dependent on Nugent scoring, where

265 recurrent patients were described as women who responded to treatment but exhibited a

266 second episode of BV during the 10-week period (Table S2). Results resonated with

267 model predictions where individuals who experienced recurrence had higher amounts of

268 Lactobacillus spp. relative to BV-associated bacteria (lower BV:LB ratios, $\mathrm{P}=0.0366$ ) and

269 tended to have higher abundances of Lactobacillus spp., particularly $L i$, but abundance 
270 of individual species were not statistically significant after adjustment for multiple

271 comparisons ( $P=0.109$, Fig. 5a fig. S8a). Additionally, Gv relative abundance was not

272 significantly different between groups $(P=0.984$, fig. S8b). Furthermore, when we

273 analyzed the specific species in the original two-species model, we also observed similar

274 results where cured women had significantly higher ratios of Gv to $L i$ ( $p=0.0249$, Fig.

275 6b). In is important to note that since the Gv:Li ratio comparison was a selective analysis,

276 we did not correct for multiple comparisons based on individual species in the original

277 data set (over $\sim 190$ species measured). These results support both the in vitro

278 experimental data and model results that predicted a lower efficacy of MNZ treatment

279 when a lower ratio of Gv to Li was present pre-treatment.

280 We also evaluated model findings in a second clinical cohort, the CONRAD BV

281 study, which consisted of 33 women whose vaginal microbiome was sampled at

282 enrollment in the study, one week after MNZ treatment and one month after MNZ

283 treatment. Relative abundances were determined by sequencing of the $\mathrm{V} 4$ region of the

284 16S rRNA. Women were excluded from this subset analysis if they failed to finish

285 antibiotic regimen, contracted a secondary vaginal infection, did not respond or had

286 delayed response of treatment. Of the 33 women, 21 met inclusion criteria and were

287 evaluated by molecular-BV criteria (dominance of Lactobacillus spp.) at one week and

288 one month, with women exhibiting a vaginal microbiota composition of less than $50 \%$

289 Lactobacillus sp. classified as BV positive. The group analyzed consisted of women who

290 were cured $(n=10)$ and or were determined to have recurrent BV $(n=11$; Lactobacillus was

291 dominant at one week, but molecular-BV returned after one month, Table S3). Similar to

292 the previous study, we found that women who experienced recurrence had higher levels 
293 of Lactobacillus spp. relative to BV-associated bacteria (lower BV:LB ratio, $\mathrm{P}=0.0484$,

294 Fig. 5c). Comparison of CLR-transformed relative abundance did not result in statistically 295 significant differences for $L i$ or $G v$, but tended to support the trend of recurrent women 296 having higher $L i$ and lower Gv (fig. S8 c \& d, P = 0.526, P = 0.539). Similarly, analysis of 297 the Gv:Li ratio supported higher pre-treatment Gv relative to Li was associated with better 298 treatment outcomes (Fig. $5 d ; p=0.0191$ ). Though preliminary and limited by low sample 299 numbers, these results support the model predictions and suggest that successful BV 300 treatment could be driven by competition for MNZ, where non-target bacterial populations, 301 Lactobacillus spp., like Li sequester MNZ away from target bacterial populations like Gv, 302 A. vaginae, Sneathia, etc., ultimately decreasing MNZ efficacy.

\section{Discussion}

Here we show a new, highly personalized tolerance mechanism that may 306 contribute to BV recurrence and treatment failure. Our model illustrates how non-target 307 bacteria, such as Li or other Lactobacillus spp., may sequester antibiotic and lower the 308 amount of MNZ available to target bacteria like Gv. The potential for non-antibiotic-target 309 bacterial populations to act as a sink for MNZ and alter efficacy is similar to a concept 310 that has been previously explored in bacterial ecology and termed the "the inoculum effect

311 (IE)", which describes an increase in antibiotic MICs due to increased initial bacterial load

312 and decreased per cell antibiotic concentration. ${ }^{55}$ This model result implies that MNZ 313 efficacy may be dependent on highly variable pre-treatment relative abundances of 314 Lactobacillus spp. such as $L i$ to BV-associated bacteria populations (BV:LB ratios) and 315 raises the question of whether patients with higher levels of Lactobacillus spp. are more 
susceptible to recurrent BV than those with higher degrees of dysbiosis. Importantly,

317 results from the model, in vitro experiments, and clinical data all point to a higher pre-

318 treatment BV-associated bacteria population relative to Lactobacillus spp. as a driver of

319 MNZ efficacy in inhibiting Gv growth and facilitating post-treatment Lactobacillus-

320 dominance. This study complements ongoing work in the search for drivers of BV

321 treatment efficacy, in which experimental studies are often limited to delineating the role

322 of individual bacteria, and it is challenging to assess the importance of numerous clinical

323 and microbial variables that are associated with treatment outcome. ${ }^{15}$

324 Recent studies evaluating pre-treatment vaginal microbiota composition on MNZ

325 efficacy have reported inconsistent results, likely due to differences in patient exclusion

326 criteria, timepoint of treatment outcome assessment, drug regimen, and methods to

327 collect and quantify the vaginal microbiota. One study that employed a similar drug

328 regimen (oral MNZ) and sample collection methods to the clinical cohorts evaluated here

329 supported our results, finding higher pre-treatment loads of antibiotic-target species, Gv

330 and $A$. vaginae, associated with BV treatment efficacy. ${ }^{19}$ Other studies that used different

331 sample collection methods and antibiotic regimens did not explicitly evaluate the pre-

332 treatment ratio of BV-associated bacteria to Lactobacillus spp.; generally suggested there

333 was an association between total Lactobacillus relative abundance and successful

334 treatment. ${ }^{15,54,56}$ Notably, some of these studies focused on analyzing treatment outcome

335 immediately after antibiotic therapy was completed, and in some cases treatment failure

336 was due to no response to therapy. We propose that recurrence and failure to respond to

337 therapy likely arise from different factors, where recurrence is due to a collective bacterial

338 population's resilience to antibiotic therapy and failure to respond is due to inherent 
339 resistance of BV-associated bacteria. Studies that have associated higher Gv loads with

340 treatment failure correspond with the latter and could be due to the formation of biofilms

341 or other resistance mechanisms. ${ }^{56}$ As our model predicts immediate post-therapy

342 Lactobacillus spp. relative abundance, no response to treatment would be equivalent to

343 predicting no change or low Lactobacillus spp. relative abundance at $48 \mathrm{~h}$. An additional

344 limitation of our model is that it does not appear to be applicable to cases of MNZ

345 treatment failure in women who initially had very low levels of Lactobacillus sp. $(<1 \%)$,

346 which our model would predict should promote MNZ efficacy. ${ }^{34}$ However, we propose that

347 treatment failure in this case may be a result of the Allee effect, ${ }^{62,63}$ which can be caused

348 by a variety of mechanisms that lead to decreased fitness at low population densities,

349 suggesting these women have Lactobacillus abundances that are too low to recolonize

350 the vagina and may be associated with more precisely modeling inter-species

351 interactions. Moreover, since $L i$ is the only Lactobacillus sp. observed to date to

352 significantly sequester MNZ, it will be important to characterize how other vaginal

353 bacterial species interact with MNZ to further explore the role of non-target bacterial

354 species on MNZ efficacy. Altogether, conflicting results in clinical studies of pre-treatment

355 vaginal microbiota composition support the need for the development of quantitative

356 platforms to evaluate the interplay between multiple microbial species, clinical variables,

357 and dosing regimens that contribute to personalized differences in treatment failure.

$358 \quad$ Models presented here are only simple reconstructions of the minimal possible 359 interactions between bacterial species and an antibiotic that have been established as 360 key species by the existing literature ${ }^{10,15,17}$, with a time-scale that was limited by in vitro 361 co-culture conditions. While the model provided striking insight into how non-target 
362 bacterial species may influence BV recurrence after MNZ treatment, predicting regrowth

363 of Lactobacillus spp., and the full quantitative mechanisms underlying responses to

364 treatment are likely more nuanced. More complex model frameworks did suggest key

365 results would hold true in microbial communities with additional microbial species,

366 interspecies interactions, and strain variability, though we were not able to validate this

367 experimentally. Interspecies interactions in our models were incorporated with

368 generalized Lotka-Volterra equations which simplifies relationships to a single term, but

369 represent a good starting point for recapitulating ecosystem-level complexities. ${ }^{57,58,59-61}$

370 Specific metabolic interactions that dictate survival and elimination of bacterial species in

371 the vagina could be included with greater mechanistic detail in the future. In instances

372 where parameters are unknown or difficult to measure experimentally, this work

373 demonstrates the value of a global computational sensitivity analysis for understanding

374 the relative importance of strain-level differences in antibiotic uptake, metabolism, or

375 sensitivity. Predictive simulations can be run across multiple possible parameter ranges

376 to determine the effects of variation prior to costly experimental measurements. This tool

377 will be valuable in isolating the role of individual parameters in making a bacterial

378 population or community more tolerant to antibiotic therapy.

379 In this study we demonstrated that ODE models can provide insights into antibiotic-

380 microbe interactions pertinent to understanding BV treatment efficacy. Our work

381 highlights that it is possible for BV treatment to fail, even if target bacteria are not resistant

382 to MNZ as vaginal bacterial populations as a whole can be resilient to antibiotic, resulting

383 in recurrent BV. While our clinical analysis is limited in sample size and therefore should

384 be considered preliminary, future extensions of this work could be used to inform clinical 
385 decision-making regarding personalized therapy options. More generally, we envision

386 that the use of quantitative models such as this will provide a framework for integrating

387 knowledge of interactions between multiple bacterial species and drug treatments in

388 mucosal tissues to give new insight into the diverse responses observed in infectious 389 disease and other syndromes of the female reproductive tract.

\section{Methods}

392 Bacterial Strains and Culture Conditions

393 Lactobacillus iners ATCC 55195 and Gardnerella vaginalis ATCC 14018 (group C) were 394 obtained from the American Type Culture Collection (ATCC) and maintained on Human 395 Bilayer Tween Agar (BD) plates and New York City III (NYCIII) medium according to the 396 manufacturer's instructions. Agar plates and liquid cultures were incubated at $37^{\circ} \mathrm{C}$ with 397 anaerobic gas mixture, $80 \% \mathrm{~N}_{2}, 10 \% \mathrm{CO}_{2}$, and $10 \% \mathrm{H}_{2}$. Frozen stocks of strains were 398 stored at $-80^{\circ} \mathrm{C}$ in $40 \%(\mathrm{v} / \mathrm{v})$ glycerol.

399 Metronidazole Quantification by Tandem Mass Spectrometry

400 MNZ concentrations were determined by validated LC-MS/MS assays. Sample aliquots 401 were centrifuged at $3000 \times G$ and divided between supernatant and cell pellet. Extracellular 402 MNZ was extracted from supernatant via protein precipitation using acetonitrile. For intra403 cellular concentration measurements, cell pellets were lysed using sonication and re404 suspended in $100 \mu \mathrm{L}$ of sterile water. Samples were subjected to positive electrospray 405 ionization (ESI) and detected via multiple reaction monitoring (MRM) using a LC-MS/MS 406 system (Agilent Technologies 6460 QQQ/MassHunter). Calibration standards were 
407 prepared with an inter- and intra-day precision and accuracy of $\leq 5 \%$ with an $r^{2}$ value of

$4080.9988 \pm 0.0009$. Quantification was performed using MRM of the transitions of $\mathrm{m} / \mathrm{z}$ $409 \quad 172.2 \rightarrow 128.2$ and $176.2 \rightarrow 128.2$ for MNZ and MNZ-d4 respectively. Each transition was 410 monitored with a 100-ms dwell time. Stock solutions of MNZ and MNZ-d4 were prepared 411 at $1 \mathrm{mg} / \mathrm{mL}$ in acetonitrile-water and stored at $-20^{\circ} \mathrm{C}$. Mobile phase $A$ is $0.1 \%$ acetic acid 412 in $\mathrm{H}_{2} \mathrm{O}$ and mobile phase $\mathrm{B}$ is $0.1 \%$ acetic acid in $\mathrm{ACN}$, and chromatographic separation 413 was achieved using a gradient elution with a Chromolith Performance RP-C18 column 414 maintained at $25^{\circ} \mathrm{C}$ from $0-4.6$ minutes, $\mathrm{B} \% 0-100$, with $0.5 \mu \mathrm{L} / \mathrm{min}$ flow. During pre-study 415 validation, calibration curves were defined in multiple runs on the basis of triplicate assays 416 of spiked media samples as well as QC samples. This method was validated for its

417 sensitivity, selectivity, accuracy, precision, matrix effects, recovery, and stability.

418 Replicates of reference samples were included every 6 samples and evenly distributed 419 throughout the MS analysis to monitor consistency and performance and to utilize for 420 downstream normalization.

\section{Bacterial Quantification}

422 Bacterial quantification determined via turbidimetry was completed by measuring the 423 optical density at each time point, $100 \mu \mathrm{L}$ of sample inoculum was read at O.D. $600 \mathrm{~nm}$ 424 using a SpectraMax Plus 384 UV spectrophotometer. Time points were recorded within 4255 minutes of sampling and stored at $4^{\circ} \mathrm{C}$.

426 Bacterial quantification using plate counting was done by doing a 10 -fold dilution using 427 sterile water and aliquoting $100 \mu \mathrm{L}$ spread evenly onto BD agar plates. Cultures were 428 incubated at $37^{\circ} \mathrm{C}$. Plating was done in triplicates and were counted manually. Prior 
429 optimization ensured the dilution would result in no more than 300 colonies making 430 quantification as accurate as possible.

431 For co-culture validation experiments, $100 \mathrm{uL}$ of sample was aliquoted on Rogosa agar

432 and Gardnerella selective agar. Experiments were conducted to verify absence of

433 Lactobacillus growth on Gardnerella selective media and absence of $G$. vaginalis growth

434 on Rogosar agar, to confirm that colony formation specific to respective taxa. Cultures

435 were incubated at $37^{\circ} \mathrm{C}$, with a total of 36 biological replicates for the $1,000 \mathrm{x}$ and 0.001

436 Gv:Li ratio cultures ( $\mathrm{n}=18$ cultures for each ratio). Plating was done in triplicates and

437 were counted manually. Prior optimization ensured the dilution would result in no more

438 than 300 colonies making quantification as accurate as possible.

\section{Bacteria-MNZ experiments}

440 For the MNZ experiments, $50 \mu \mathrm{L}$ MNZ was added at appropriate concentrations to $5 \mathrm{~mL}$ of

441 NYCII media. Samples equilibrated at $37^{\circ} \mathrm{C}$ for 1 hour prior to the addition of $50 \mathrm{uL}$ of

442 bacterial inoculum $\left(2 \times 10^{6}\right) .150 \mu \mathrm{L}$ aliquot was taken for time point readings for MNZ and

443 bacterial quantification (as described above). Samples were incubated at $37^{\circ} \mathrm{C}$ under 444 constant mixing and only removed for time point measurements.

445 For the co-culture experiments, Gv:Li ratios were added at appropriate experimental 446 conditions in a like-wise manner. For each varying ratio sample within each experiment, 447 a side-by-side duplicate was performed without MNZ as a negative control. The negative 448 control was assessed only for bacterial quantification to ensure that no growth condition 449 or external stimuli promoted the growth of one over another. Negative control experiments 450 demonstrated bacterial proliferation that modelled growth curves of each individual 
451 bacterium cultured alone thus confirming any changes in growth seen in our bacteria-

452 MNZ experiments were the result of the addition of MNZ.

\section{ODE Models.}

454 The model equations were constructed assuming both Li and Gv internalize MNZ at rates

455 kint-Li and kint-Gv, MNZ toxicity to $L i$ and Gv occurred at rates dependent on the maximum

456 rates $k_{\text {kill-Li }}$ and $k_{\text {kill-Gv }}$ and the concentration of internalized MNZ where growth inhibition

457 increased as internalized MNZ exceeded a threshold as described by $50 \%$ effective 458 concentrations, EC50Li and EC50Gv. The growth of Li and Gv was assumed to be logistic

459 in behavior at rates kgrow-Li and kgrow-Gv with distinct carrying capacities for each bacterium, $460 \mathrm{~K} \mathrm{~K}_{\mathrm{Li}}$ and $\mathrm{K}_{\mathrm{Gv}}$ The parameters for Kgrow-Li, Kgrow-Gv, $\mathrm{K}_{\mathrm{Li}}$ and $\mathrm{K}_{\mathrm{Gv}}$ were determined by nonlinear

461 least squares fitting of the logistic function to growth curves for $L i$ and Gv grown in 462 separate cultures (fig. S2a \& b). ${ }^{65}$ The kkill-Li, $\mathrm{k}_{\text {kill-Gv }}$ EC50 Li and EC50Gr where determined 463 by fitting the Hill equation to kill curves for Li and Gv cultured in isolation (fig. S2c-e). 464 Internalization rates, $k_{\text {int-Li }}$ and kint-Gv and metabolism rates, kacet and kmet were determined 465 from fitting the ODE model to time course mass spectrometry data for external MNZ, 466 internal MNZ and acetamide and cell densities (Optical density) using a multi-start local 467 optimization strategy (Multistart) with the local solver Isqcurvefit.

\section{Model Simulations and Validation.}

469 Unless otherwise noted, all simulations were completed at MNZ concentration of 500 $470 \mu \mathrm{g} / \mathrm{ml}$ over the course of $48 \mathrm{~h}$. Growth outputs were normalized to the maximal growth 471 density ( $\mathrm{K}_{\mathrm{Li}}$ and $\mathrm{K}_{\mathrm{GV}}$ ) for comparison across simulations and to experimental data. 472 External MNZ, internal MNZ and acetamide concentrations were relative to the total 
473 volume of cellular pellet. Sensitivity analyses were completed by perturbing a single

474 model parameter while keeping the rest of the parameters constant over $1,000 \mathrm{x}-0.001 \mathrm{x}$

475 the original value. Surfaces were generated over three orders of magnitude for MNZ

476 concentration $(10-1,500 \mu \mathrm{g} / \mathrm{ml})$ and eight orders of magnitude for ratio of Gv:Li (1.6x10-

$4774^{4}-1.6 \times 10^{4}$ ) at 1225 combinations of MNZ concentration and Gv:Li ratio. Model validation

478 was completed by comparing the experimental co-culture data to model predictions using

479 unpaired t-tests.

480 Generalized Models, inter- and intra-Species Variability and Global Sensitivity Analysis

481 To incorporate intraspecies and interspecies variation we developed three additional

482 model structures and ran simulations with randomized parameter sets to determine if the

483 influence of initial Gv:Li ratio, or the more generalized BV:LB ratio, on endpoint

484 Lactobacillus spp. composition is consistently observed across these model structures.

485 For capturing intraspecies variation, we used Latin Hypercube Sampling of parameter 486 ranges for each parameter to create 100 parameter sets. We derived these parameter

487 ranges from the literature and a summary of these ranges can be found in Table S2 and 488 Table S3. These same parameter ranges and sampling method were used for the global 489 sensitivity and uncertainty analysis, which analyzed the partial rank correlation coefficient 490 with 2,000 randomly generated parameter sets with endpoint (48h, $500 \mu \mathrm{g} / \mathrm{ml} \mathrm{MNZ)}$

491 Lactobacillus spp. relative abundance (Marino et al., 2008). ${ }^{66}$ For capturing interspecies 492 variation, and microbe-microbe interactions like cross-feeding, we developed a four 493 species model that includes two representative BV-associated bacteria, and two 
494 Lactobacillus species, L. iners and a second species representing L. crispatus, L. jensenii, 495 or L. gasseri.

496 Internalization/Uptake Rates (kint): To our knowledge, this is the first publication that 497 demonstrates that $G$. vaginalis and $L$. iners uptake or sequester MNZ. Previous literature 498 describing uptake of MNZ in other bacterial species, including both obligate and 499 facultative anaerobes has been published by Ralph and Denise Clarke (1978), Tally et al 500 (1978) and Narikawa (1986). ${ }^{27,31,67}$ These publications demonstrate that even bacteria 501 that are resistant to MNZ can still uptake MNZ, and at similar rates. Despite the fact that 502 facultative anaerobes are believed to be largely insensitive to MNZ, Narikawa specifically 503 demonstrates that nitroreductase activity is associated with the ability to uptake MNZ, and 504 that pyruvate:ferrodoxin activity is associated with sensitivity to MNZ as an explanation 505 for why the facultative anaerobes Escherichia coli, K. pneumoniae, M. morganii and S. 506 faecalis exhibited high MICs, but reduced supernatant MNZ. We calculated the rates of 507 MNZ uptake for five species, one obligate anaerobe, $B$. fragilis, and four facultative 508 anaerobes (E. coli, S. aureus, P. morganii and S. faecalis) by digitizing the kinetic data 509 for cell counts and extracelluar MNZ concentrations in Ralph and Denise Clarke (1978) 510 and fitting second order reaction kinetics by ordinary least squares regression. The rates 511 ranged from $2 \times 10^{-17}$ to 0.15 cell density ${ }^{-1} \mathrm{~h}^{-1}$. To determine the likelihood that these

512 parameters could be a basis for Lactobacillus spp. uptake of MNZ, we assessed the 513 similarity between $E$. coli's oxygen independent NADPH-nitroreductase, nsfA, with 514 nitroreductase protein sequences of G. vaginalis (34.7\%), L. crispatus (31.0\%), L. iners 515 (29.4\%), L. jensenii (19.4\%) and L. gasseri (18.52\%). Additionally, Guillen et al (2009) 516 reported that L. plantarum had selective nitroreductase activity, that shared $32-43 \%$ 
517 sequence similarity with several Lactobacillus species, and in comparison had similarity

518 with G. vaginalis (24.0\%), L. crispatus (38.5\%), L. iners (25.5\%), L. jensenii (52.8\%) and

519 L. gasseri (30.0\%). ${ }^{68}$ Sequence similarity was assessed by NCBI's protein BLAST. ${ }^{69}$ As

520 obligate anaerobes were observed to uptake MNZ at higher rates, we assumed that the

521 other BV-associated bacteria, which could be an obligate anaerobe could potentially have

522 higher capacity to internalize MNZ.

523 Growth Rates (kgrow) and Carrying Capacities (K): To take into account potential variability

524 in growth rates, we surveyed previously published to determine ranges in growth. For

525 Lactobacillus species, we calculated growth rates by digitizing growth curves from

526 Chetwin et al (2019) and analyzed growth rates reported in Tomas (2003), Anukam and

527 Reid (2008). G. vaginalis and other bacterial strains growth curves were less abundant in

528 the literature, but we did calculate growth rates from Atassi et al., 2019 and Anukam and

529 Ried (2008). ${ }^{45,49,70,71}$ Generally, G. vaginalis and other BV-associated bacteria seemed to

530 have slower growth rates than Lactobacillus species, and in the same culture conditions

531 this was observed in Anukam and Ried (2008). For carrying capacity we assumed that

532 there were similar carrying capacities for all species, except the BV-associated bacteria

533 based on data from Castro et al 2020, that reported $A$. vaginae at lower levels that $G$.

534 vaginalis at steady state. ${ }^{72}$

535 Sensitivity to MNZ (EC50 and kkill): MNZ is highly variable, and typically obligate

536 anaerobes are considered the most sensitive to MNZ. The strain of $G$. vaginalis used in

537 the basis of this model is relatively resistant to MNZ, with growth barely inhibited at 256

$538 \mu \mathrm{g} / \mathrm{ml}$ (9\% inhibition compared to $0 \mu \mathrm{g} / \mathrm{ml}$ control, Fig. S2). For $A$. vaginae, the MIC can

539 range $2 \mathrm{ug} / \mathrm{ml}-256 \mathrm{ug} / \mathrm{ml}$ and $\mathrm{G}$. vaginalis can range from $0.75 \mu \mathrm{g} / \mathrm{ml}$ to $>500 \mu \mathrm{g} / \mathrm{ml} .43,44$ 
540 Generally, it is assumed that Lactobacillus spp. are insensitive to MNZ; however, this also

541 appears to be highly strain and species dependent with some Lactobacillus isolates in

542 similar ranges of sensitivity as G. vaginalis. ${ }^{42,45}$ The rate at which MNZ inhibits growth is

543 more difficult to find, as the experiments to determine this rate are more laborious than

544 the standard kill curve to calculate EC50 so we assumed all kill rates to be equal across

545 all species.

546 Metabolism of MNZ: To our knowledge, this is the first manuscript to describe the

547 metabolism of MNZ by vaginal microbiota. We solely based the parameter value on the

548 rate observed for the $G$. vaginalis strain from the model. Additionally, we assumed that

549 only BV-associated bacteria metabolism MNZ based on the observation that only BV-

550 associated bacteria metabolize HIV microbicide drugs. ${ }^{29}$

551 Inter-species Interaction Terms: Gause (1934) first noted the calculation for interaction

552 terms for a generalized Lotka-Volterra model describing competitive exclusion (equation

5531 and 2). In our model, we generalized the interaction terms further to be able to capture

554 many different interactions, specifically amensal behavior where Lactobacillus spp. can

555 inhibit BV-associated bacterial growth with no effect of BV-associated bacteria on

556 Lactobacillus species growth (-/0) as well as mutualistic (both species benefit from the

557 other $+/+$ ) and commensal behaviors (one species benefits $0 /+$ ) between $B V$-associated

558 bacteria or within the Lactobacillus population. The amensal behavior between

559 Lactobacillus species has been documented experimentally in co-culture (Jackman et al.,

$5602019)$ and we calculated the interaction term for many different species and strains of

561 Lactobacillus on G. vaginalis and Prevotella bivia from Atassi et al. (2006). ${ }^{50,73}$ It is largely

562 believed that D-lactic acid produced by many Lactobacillus species inhibits the growth of 
563 BV-associated bacteria; however, L. iners does not produce this isomer of lactic acid and

564 is the reasoning behind not including an interaction term between $L$. iners and the BV-

565 associated bacteria. ${ }^{48,74}$ It is believed that commensal behavior exists between $G$.

566 vaginalis and $P$. bivia in the form of cross-feeding, so we allowed the model to simulate

567 this behavior. ${ }^{51}$ Additionally, G. vaginalis is associated with promoting the growth of other

568 BV-associated bacteria like $A$. vaginae. ${ }^{72}$ Calculations were completed assuming the

569 reported mono and co-cultures were at steady state to derive equation 3 and 4 . Equations

5702 and 3 relate to the parameter table S3 in equations 5 and 6 , which generalizes the

571 reported interaction strength from the literature to be able to be adjusted for varying

572 carrying capacities simulated in the model that do not equal the carrying capacities from

573 the literature.

574

$$
\text { 1. } \frac{d N}{d t}=r_{\mathrm{N}} N\left[1-\frac{\mathrm{N}+\boldsymbol{s}_{\mathrm{P} \rightarrow \mathrm{N}} P}{K_{\mathrm{N}}}\right] \quad 2 \cdot \frac{d P}{d t}=r_{\mathrm{P}} P\left[1-\frac{P+s_{\mathrm{N} \rightarrow \mathrm{P}} \boldsymbol{N}}{K_{\mathrm{P}}}\right]
$$

575

$$
\text { 3. } \boldsymbol{s}_{\mathrm{P} \rightarrow \mathrm{N}}=\left[\frac{K_{\mathrm{N}}-\mathrm{N}}{P}\right] \quad \text { 4. } \boldsymbol{s}_{\mathrm{N} \rightarrow \mathrm{P}}=\left[\frac{K_{\mathrm{P}}-\mathrm{P}}{N}\right]
$$

576

577

$$
\text { 5. } \boldsymbol{s}_{\mathrm{P} \rightarrow \mathbf{N}}=\left[\frac{K_{\mathrm{N}}-f_{\mathrm{P} \rightarrow \mathrm{N}} K_{\mathrm{N}}}{K_{\mathrm{P}}}\right] \quad \text { 6. } \boldsymbol{s}_{\mathrm{N} \rightarrow \mathrm{P}}=\left[\frac{K_{\mathrm{P}}-\boldsymbol{f}_{\mathrm{N} \rightarrow \mathrm{P}} K_{\mathrm{P}}}{K_{\mathrm{N}}}\right]
$$

578 Software. Parameterization, ODE modeling, sensitivity analyses, and PLSDA were

579 completed using Matlab® 2018b (Matlab, Natick, MA). Statistical analyses were 580 performed using PRISM 8.

\section{Clinical data and study population}

582 The UMB-HMP cohort: The study results and associated clinical data were previously

583 published (Ravel et al., 2013) and all data provided was de-identified to this study. The 
584 original clinical study protocol was approved by the Institutional Review Board of the 585 University of Alabama at Birmingham and the University of Maryland School of Medicine.

586 Written informed consent was appropriately obtained from all participants, who 587 also provided consent for storage and used in future research studies related to women's 588 health. A total of 135 nonpregnant women of reproductive age were enrolled in a 589 longitudinal study at the University of Alabama at Birmingham. Vaginal microbiota data 590 was generated by sequencing the V3-V4 regions of the $16 \mathrm{~S}$ rRNA gene and is available 591 at in dbGAP BioProject PRJNA208535.

592 Women self-collected cervicovaginal swabs for 10 weeks. The vaginal microbiota 593 composition data, previously established by sequencing of the V3-V4 regions of the $16 \mathrm{~S}$ 594 rRNA gene, from 11 women who experienced BV (one week twice daily $500 \mathrm{mg}$ oral MNZ) 595 and were treated during the UMB-HMP study were analyzed. Any participants who failed 596 to complete the MNZ regimen, who did not have BV according to Nugent scoring at the 597 time of MNZ treatment, or who did not have follow-up data available were excluded from 598 the analysis. The initial ratios of Gv:Li relative abundances were averaged across the 599 week before starting MNZ treatment. Patients were classified to have recurrent BV if they 600 exhibited a second episode of BV based on Nugent scoring (7-10) during remaining of 601 the 10-week observation period.

602 The CONRAD BV cohort: The study results and associated clinical data were previously 603 published (Thurman et al., 2015) and all data provided was de-identified to this study. The 604 original clinical study protocol was approved by the Chesapeake Institutional Review 605 Board (IRB) (Pro \#00006122) with a waiver of oversight from the Eastern Virginia Medical 606 School (EVMS) and registered in ClinicalTrials.gov (\#NCT01347632). A total of 69 
607 women were screened from symptomatic discharge and 35 women were enrolled in the

608 study. Vaginal microbiota data was generated by sequencing the V4 region of the 16s

609 rRNA gene, providing taxonomic resolution at the genera level.

610 Thirty-three women completed all three visits. BV was evaluated by vaginal microbiota

611 compositional data (molecular-BV). ${ }^{64}$ After biological samples were obtained at visit 1

612 (V1), women with BV were prescribed twice daily, 500-mg MNZ for 7 days. Participants

613 returned for visit 2 (V2) 7-10 days after completing the course of MNZ therapy and visit 3

614 (V3) 28-32 days after completing treatment. At all three visits samples were obtained to

615 evaluate vaginal semen (ABAcard, West Hills, $\mathrm{Ca}$ ), vaginal $\mathrm{pH}$, gram stain for Nugent

616 score and semiquantitative vaginal flora culture. CVLs were collected, followed by vaginal

617 swabs and three full-thickness biopsies.

\section{Analysis of Clinical Outcomes.}

619 In the Human Microbiome Project cohort, patients were defined as cured or recurrent

620 based on whether after initial MNZ treatment the patient suffered an additional episode

621 of BV (Nugent 7-10) during the 10-week course of data collection. For analysis, initial flora

622 relative abundances were averaged across the 7 days prior to reported treatment start

623 date. To analyze the relative ratio between BV-associated bacteria and Lactobacillus

624 spp., we combined the relative abundances for the top twenty BV-associated bacteria

625 and all Lactobacillus spp. The genera BV-associated bacteria included were: Gardnerella,

626 Atopobium, Megasphaera, BVAB1-3, Streptococcus, Prevotella, Leptotrichia,

627 Anaerococcus, Peptoniphilus, Eggerthella, Veillonella, Sneathia, Mobiluncus,

628 Corynebacterium, Ureaplasma, Eubacterium, Porphyromonas, Dialister,

629 Peptostreptococcus, Bacteroides, Fusobacterium, Actinomyces, Bifidobacterium. Before 
630 statistical analysis, the BV:LB ratio was log-transformed, and the relative abundances of

631 L. iners, G. vaginalis were center-log ratio (CLR) transformed, with pseudocounts added

632 to taxonomic units with relative abundances equal to zero, to compare between cured 633 and the recurrent groups by one-way unpaired t-tests and were corrected using the FDR 634 method of Benjamini and Hochberg (PRISM 8).

635 For the CONRAD BV cohort, treatment outcome was defined based on Lactobacillus 636 dominance evaluated at enrollment, 7 days after treatment and 28-32 days after 637 treatment. Patients that exhibited Lactobacillus dominance at both 1 week and 1 month 638 after treatment were considered cured, and patients that exhibited Lactobacillus 639 dominance only at week 1 and not at 1 month were considered recurrent. The statistical 640 analysis followed the same methodology as the HMP Cohort.

\section{Data availability}

642 Data used to parameterize and validate the model are available upon request (Fig. 2, Fig. 643 S1-S2). The UMB-HMP cohort study sequence data and metadata were deposited in the 644 Sequence Read Archive (SRA; http://www.ncbi.nlm.nih.gov/Traces/sra/) under 645 BioProject PRJNA208535 ("The daily dynamics of the vaginal microbiota before and after 646 bacterial vaginosis"; http://www.ncbi.nlm.nih.gov/bioproject/? term=PRJNA208535) 647 ([SRP026107] and [SRA091234]). An abbreviated data set necessary for the reproduction 648 of Fig. 5a-c is in Table S4. The data from the CONRAD BV study are not in a formal 649 repository, but are fully available upon request. An abbreviated data set necessary for 650 reproduction of Fig. $5 d-f$ is in Table S5. 
652 Code availability

653 All code is available at https://github.com/chyylee/BV.

\section{Competing Interests}

655 JR is co-founder of LUCA Biologics, a biotechnology company focusing on translating 656 microbiome research into live biotherapeutic drugs for women's health. All other authors 657 declare no competing interests.

658

\section{Authorship Contributions}

$660 \mathrm{CYL}, \mathrm{RKC}, \mathrm{MML}, \mathrm{NRK}$ and KBA conceived and designed the study. CYL completed the 661 computational analysis and analyzed the clinical data. RKC, NRK, and BH designed and 662 conducted monoculture and co-culture kinetic experiments. AG, MF, and JR curated data 663 from the UMB-HMP cohort. JR lead the UMB-HMP study and data collection. AT and GD 664 provided CONRAD BV protocol development, patient care, and data analysis. CYL, RKC, $665 \mathrm{KBA}$, and NRK wrote the manuscript and all authors read and revised the manuscript.

\section{Acknowledgements}

668 This work was in part funded by startup funds from the University of Miami to NRK, funds

669 from NIH/NIDDK grant RO1DK112254 to NRK, and NIH/NIAID grant R01Al138718

670 subcontract to NRK, and startup funds from the University of Michigan to KBA. The UMB-

671 HMP study, JR and MF were supported by the National Institute for Allergy and Infectious 
672 Diseases of the National Institutes of Health under award numbers UH2AI083264 and

673 R01NR015495. The CONRAD BV Study was funded by an intra-agency agreement

674 between the Centers for Disease Control and Prevention (CDC), United States Aid and

675 International Development (USAID) and CONRAD (GPO-A-00-08-00005-00). The views

676 expressed by the authors do not necessarily reflect those of the funding agency or

677 CONRAD.

\section{References}

1. Koumans, E. et al. The Prevalence of Bacterial Vaginosis in the United States, 20012004; Associations With Symptoms, Sexual Behaviors, and Reproductive Health.

Sex. Transm. Dis. 34, 864-869 (2007).

2. Peebles, K., Velloza, J., Balkus, J. E., McClelland, R. S. \& Barnabas, R. V. High Global Burden and Costs of Bacterial Vaginosis: A Systematic Review and MetaAnalysis. Sex. Transm. Dis. 46, 304-311 (2019).

3. Taha, T. et al. Bacterial vaginosis and disturbances of vaginal flora: association with increased acquisition of HIV. Aids 12, 1699-1706 (1998).

4. Haggerty, C. L., Hillier, S. L., Bass, D. C. \& Ness, R. B. Bacterial Vaginosis and Anaerobic Bacteria Are Associated with Endometritis. Clin. Infect. Dis. 39, 990-995 (2004).

5. Hillier, S. L. et al. Association between bacterial vaginosis and preterm delivery of a low-birth-weight infant. The Vaginal Infections and Prematurity Study Group. N. Engl. J. Med. 333, 1737-1742 (1995). 
6. Bradshaw, C. S. \& Brotman, R. M. Making inroads into improving treatment of bacterial vaginosis - striving for long-term cure. BMC Infect. Dis. 15, 292 (2015).

7. Ness, R. B. et al. A cluster analysis of bacterial vaginosis-associated microflora and pelvic inflammatory disease. Am. J. Epidemiol. 162, 585-590 (2005).

8. Nunn, K. L. \& Forney, L. J. Unraveling the Dynamics of the Human Vaginal Microbiome. Yale J. Biol. Med. 89, 331-337 (2016).

9. Fredricks, D. N., Fiedler, T. L., Thomas, K. K., Mitchell, C. M. \& Marrazzo, J. M. Changes in Vaginal Bacterial Concentrations with Intravaginal Metronidazole Therapy for Bacterial Vaginosis as Assessed by Quantitative PCR. J. Clin. Microbiol. 47, 721726 (2009).

10. Srinivasan, S. et al. Temporal Variability of Human Vaginal Bacteria and Relationship with Bacterial Vaginosis. PLOS ONE 5, e10197 (2010).

11. Bradshaw, C. S. et al. High Recurrence Rates of Bacterial Vaginosis over the Course of 12 Months after Oral Metronidazole Therapy and Factors Associated with Recurrence. J. Infect. Dis. 193, 1478-1486 (2006).

12. Bradshaw, C. S. \& Sobel, J. D. Current Treatment of Bacterial VaginosisLimitations and Need for Innovation. J. Infect. Dis. 214 Suppl 1, S14-20 (2016).

13. Ma, L., Su, J., Su, Y., Sun, W. \& Zeng, Z. Probiotics administered intravaginally as a complementary therapy combined with antibiotics for the treatment of bacterial vaginosis: a systematic review protocol. BMJ Open 7, e019301 (2017).

14. Ferris, D. G., Litaker, M. S., Woodward, L., Mathis, D. \& Hendrich, J. Treatment of bacterial vaginosis: a comparison of oral metronidazole, metronidazole vaginal gel, and clindamycin vaginal cream. J. Fam. Pract. 41, 443-449 (1995). 
15. Xiao, B. et al. Association Analysis on Recurrence of Bacterial Vaginosis Revealed Microbes and Clinical Variables Important for Treatment Outcome. Front. Cell. Infect. Microbiol. 9, (2019).

16. Brotman, R. M. et al. A Longitudinal Study of Vaginal Douching and Bacterial Vaginosis_A Marginal Structural Modeling Analysis. Am. J. Epidemiol. 168, 188-196 (2008).

17. Wang, B. et al. Molecular analysis of the relationship between specific vaginal bacteria and bacterial vaginosis metronidazole therapy failure. Eur. J. Clin. Microbiol. Infect. Dis. 33, 1749-1756 (2014).

18. Zhou, X. et al. Recent Advances in Understanding the Microbiology of the Female Reproductive Tract and the Causes of Premature Birth. Infectious Diseases in Obstetrics and Gynecology https://www.hindawi.com/journals/idog/2010/737425/abs/ (2010) doi:10.1155/2010/737425.

19. Ferreira, C. S. T. et al. Treatment failure of bacterial vaginosis is not associated with higher loads of Atopobium vaginae and Gardnerella vaginalis. J. Med. Microbiol. 66, 1217-1224 (2017).

20. Coleman, J. S. \& Gaydos, C. A. Molecular Diagnosis of Bacterial Vaginosis: an Update. J. Clin. Microbiol. 56, (2018).

21. Gajer, P. et al. Temporal Dynamics of the Human Vaginal Microbiota. Sci. Transl. Med. 4, 132ra52 (2012).

22. Tamarelle, J. et al. Non-optimal vaginal microbiota after azithromycin treatment for Chlamydia trachomatis infection. J. Infect. Dis. (2019) doi:10.1093/infdis/jiz499. 
23. Vaneechoutte, M. Lactobacillus iners, the unusual suspect. Res. Microbiol. 168, 826-836 (2017).

24. Faust, K., Lahti, L., Gonze, D., de Vos, W. M. \& Raes, J. Metagenomics meets time series analysis: unraveling microbial community dynamics. Curr. Opin. Microbiol. 25, 56-66 (2015).

25. Lugo-Martinez, J., Ruiz-Perez, D., Narasimhan, G. \& Bar-Joseph, Z. Dynamic interaction network inference from longitudinal microbiome data. Microbiome 7, 54 (2019).

26. Brotman, R. M., Ravel, J., Cone, R. A. \& Zenilman, J. M. Rapid fluctuation of the vaginal microbiota measured by Gram stain analysis. Sex. Transm. Infect. 86, 297302 (2010).

27. Tally, F. P., Goldin, B. R., Sullivan, N., Johnston, J. \& Gorbach, S. L. Antimicrobial activity of metronidazole in anaerobic bacteria. Antimicrob. Agents Chemother. 13, 460-465 (1978).

28. Church, D. L., Rabin, H. R. \& Lalshley, E. J. Role of hydrogenase 1 of clostridium pasteurianum in the reduction of metronidazole. Biochem. Pharmacol. 37, 1525-1534 (1988).

29. Klatt, N. R. et al. Vaginal bacteria modify HIV tenofovir microbicide efficacy in African women. Science 356, 938-945 (2017).

30. Edwards, D. I. Mechanisms of selective toxicity of metronidazole and other nitroimidazole drugs. Br. J. Vener. Dis. 56, 285-290 (1980).

31. Ralph, E. D. \& Clarke, D. A. Inactivation of Metronidazole by Anaerobic and Aerobic Bacteria. Antimicrob. Agents Chemother. 14, 377-383 (1978). 
32. Edwards, D. I., Thompson, E. J., Tomusange, J. \& Shanson, D. Inactivation of metronidazole by aerobic organisms. J. Antimicrob. Chemother. 5, 315-316 (1979).

33. Ravel, J. et al. Daily temporal dynamics of vaginal microbiota before, during and after episodes of bacterial vaginosis. Microbiome 1, 29 (2013).

34. Thurman, A. R. et al. Bacterial Vaginosis and Subclinical Markers of Genital Tract Inflammation and Mucosal Immunity. AIDS Res. Hum. Retroviruses 31, 1139$1152(2015)$.

35. Koch, R. L., Chrystal, E. J., Beaulieu, B. B. \& Goldman, P. Acetamide--a metabolite of metronidazole formed by the intestinal flora. Biochem. Pharmacol. 28, 3611-3615 (1979).

36. Regoes, R. R. et al. Pharmacodynamic functions: a multiparameter approach to the design of antibiotic treatment regimens. Antimicrob. Agents Chemother. 48, 3670-3676 (2004).

37. Meredith, H. R. et al. Applying ecological resistance and resilience to dissect bacterial antibiotic responses. Sci. Adv. 4, eaau1873 (2018).

38. Ings, R. M., McFadzean, J. A. \& Ormerod, W. E. The mode of action of metronidazole in Trichomonas vaginalis and other micro-organisms. Biochem. Pharmacol. 23, 1421-1429 (1974).

39. Davis, B., Glover, D. D. \& Larsen, B. Analysis of metronidazole penetration into vaginal fluid by reversed-phase high-performance liquid chromatography. Am. J. Obstet. Gynecol. 149, 802-803 (1984). 
40. Simoes, J. A. et al. Human Immunodeficiency Virus Type 1 Stimulatory Activity by Gardnerella vaginalis: Relationship to Biotypes and Other Pathogenic Characteristics. J. Infect. Dis. 184, 22-27 (2001).

41. Brooks, J. P. et al. Changes in vaginal community state types reflect major shifts in the microbiome. Microb. Ecol. Health Dis. 28, (2017).

42. Simoes, J. A., Aroutcheva, A. A., Shott, S. \& Faro, S. Effect of metronidazole on the growth of vaginal lactobacilli in vitro. Infect. Dis. Obstet. Gynecol. 9, 41-45 (2001).

43. De Backer, E. et al. Antibiotic susceptibility of Atopobium vaginae. BMC Infect. Dis. 6, 51 (2006).

44. Petrina, M. A. B., Cosentino, L. A., Rabe, L. K. \& Hillier, S. L. Susceptibility of bacterial vaginosis (BV)-associated bacteria to secnidazole compared to metronidazole, tinidazole and clindamycin. Anaerobe 47, 115-119 (2017).

45. Anukam, K. C. \& Reid, G. Effects of metronidazole on growth of Gardnerella vaginalis ATCC 14018, probiotic Lactobacillus rhamnosus GR-1 and vaginal isolate Lactobacillus plantarum KCA. Microb. Ecol. Health Dis. 20, 48-52 (2008).

46. Buffie, C. G. et al. Profound Alterations of Intestinal Microbiota following a Single Dose of Clindamycin Results in Sustained Susceptibility to Clostridium difficileInduced Colitis. Infect. Immun. 80, 62-73 (2012).

47. France, M. T., Mendes-Soares, H. \& Forney, L. J. Genomic Comparisons of Lactobacillus crispatus and Lactobacillus iners Reveal Potential Ecological Drivers of Community Composition in the Vagina. Appl. Environ. Microbiol. 82, 7063-7073 (2016). 
48. O'Hanlon, D. E., Moench, T. R. \& Cone, R. A. Vaginal pH and microbicidal lactic acid when lactobacilli dominate the microbiota. PloS One 8, e80074 (2013).

49. Atassi, F., Pho Viet Ahn, D. L. \& Lievin-Le Moal, V. Diverse Expression of Antimicrobial Activities Against Bacterial Vaginosis and Urinary Tract Infection Pathogens by Cervicovaginal Microbiota Strains of Lactobacillus gasseri and Lactobacillus crispatus. Front. Microbiol. 10, (2019).

50. Atassi, F., Brassart, D., Grob, P., Graf, F. \& Servin, A. L. Lactobacillus strains isolated from the vaginal microbiota of healthy women inhibit Prevotella bivia and Gardnerella vaginalis in coculture and cell culture. FEMS Immunol. Med. Microbiol. 48, 424-432 (2006).

51. Pybus, V. \& Onderdonk, A. B. Evidence for a commensal, symbiotic relationship between Gardnerella vaginalis and Prevotella bivia involving ammonia: potential significance for bacterial vaginosis. J. Infect. Dis. 175, 406-413 (1997).

52. Ravel, J. et al. Vaginal microbiome of reproductive-age women. Proc. Natl. Acad. Sci. 108, 4680-4687 (2011).

53. Deng, Z.-L. et al. Metatranscriptome Analysis of the Vaginal Microbiota Reveals Potential Mechanisms for Protection against Metronidazole in Bacterial Vaginosis. mSphere 3, (2018).

54. Xiao, B. et al. Predictive value of the composition of the vaginal microbiota in bacterial vaginosis, a dynamic study to identify recurrence-related flora. Sci. Rep. $\mathbf{6}$, (2016).

55. Brook, I. Inoculum Effect. Rev. Infect. Dis. 11, 361-368 (1989). 
56. Verwijs, M. C., Agaba, S. K., Darby, A. C. \& Wijgert, J. H. H. M. van de. Impact of oral metronidazole treatment on the vaginal microbiota and correlates of treatment failure. Am. J. Obstet. Gynecol. 222, 157.e1-157.e13 (2020).

57. Stein, R. R. et al. Ecological Modeling from Time-Series Inference: Insight into Dynamics and Stability of Intestinal Microbiota. PLOS Comput. Biol. 9, e1003388 (2013).

58. May, R. Stability and Complexity in Model Ecosystems. (2001).

59. Medlock, G. L. et al. Inferring Metabolic Mechanisms of Interaction within a Defined Gut Microbiota. Cell Syst. 7, 245-257.e7 (2018).

60. Momeni, B., Xie, L. \& Shou, W. Lotka-Volterra pairwise modeling fails to capture diverse pairwise microbial interactions. eLife 6, e25051 (2017).

61. Goldford, J. E. et al. Emergent simplicity in microbial community assembly. Science 361, 469-474 (2018).

62. Allee, W. C. Principles of animal ecology,. (Saunders Co., 1949).

63. Goswami, M., Bhattacharyya, P. \& Tribedi, P. Allee effect: the story behind the stabilization or extinction of microbial ecosystem. Arch. Microbiol. 199, 185-190 (2017).

64. McKinnon, L. R. et al. The Evolving Facets of Bacterial Vaginosis: Implications for HIV Transmission. AIDS Res. Hum. Retroviruses 35, 219-228 (2019).

65. McKendrick, A. G. \& Pai, M. K. XLV.-The Rate of Multiplication of Microorganisms: A Mathematical Study. Proc. R. Soc. Edinb. 31, 649-653 (1912). 
66. Marino, S., Hogue, I. B., Ray, C. J. \& Kirschner, D. E. A Methodology For Performing Global Uncertainty And Sensitivity Analysis In Systems Biology. J. Theor. Biol. 254, 178-196 (2008).

67. Narikawa, S. Distribution of metronidazole susceptibility factors in obligate anaerobes. J. Antimicrob. Chemother. 18, 565-574 (1986).

68. Guillén, H., Curiel, J. A., Landete, J. M., Muñoz, R. \& Herraiz, T. Characterization of a Nitroreductase with Selective Nitroreduction Properties in the Food and Intestinal Lactic Acid Bacterium Lactobacillus plantarum WCFS1. J. Agric. Food Chem. 57, 10457-10465 (2009).

69. Altschul, S. F. \& Koonin, E. V. Iterated profile searches with PSI-BLAST--a tool for discovery in protein databases. Trends Biochem. Sci. 23, 444-447 (1998).

70. Chetwin, E. et al. Antimicrobial and inflammatory properties of South African clinical Lactobacillus isolates and vaginal probiotics. Sci. Rep. 9, 1917 (2019).

71. Juárez Tomás, M. S., Ocaña, V. S., Wiese, B. \& Nader-Macías, M. E. Growth and lactic acid production by vaginal Lactobacillus acidophilus $C R L 1259$, and inhibition of uropathogenic Escherichia coli. J. Med. Microbiol. 52, 1117-1124 (2003).

72. Castro, J., Rosca, A. S., Cools, P., Vaneechoutte, M. \& Cerca, N. Gardnerella vaginalis Enhances Atopobium vaginae Viability in an in vitro Model. Front. Cell. Infect. Microbiol. 10, 83 (2020).

73. Jackman, C. M., Deans, K. W., Forney, L. J. \& Lin, X. N. Microdroplet cocultivation and interaction characterization of human vaginal bacteria. Integr. Biol. 11, 69-78 (2019). 
74. France, M. T., Mendes-Soares, H. \& Forney, L. J. Genomic Comparisons of Lactobacillus crispatus and Lactobacillus iners Reveal Potential Ecological Drivers of Community Composition in the Vagina. Appl. Environ. Microbiol. 82, 7063-7073 (2016). 


\section{FIGURES}

a

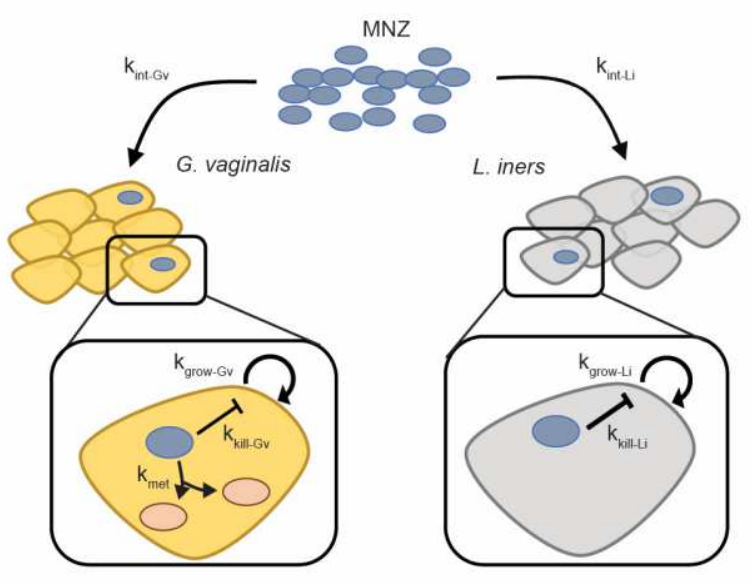

d

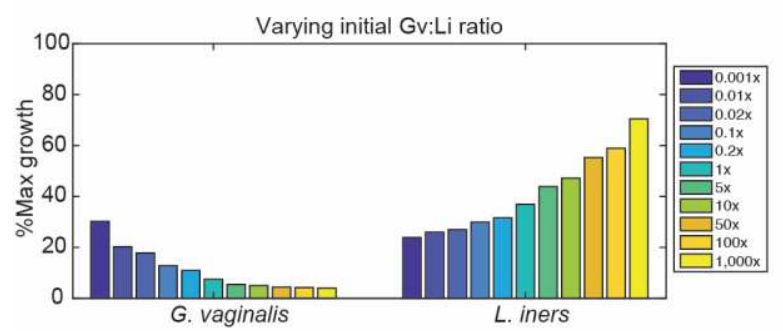

b
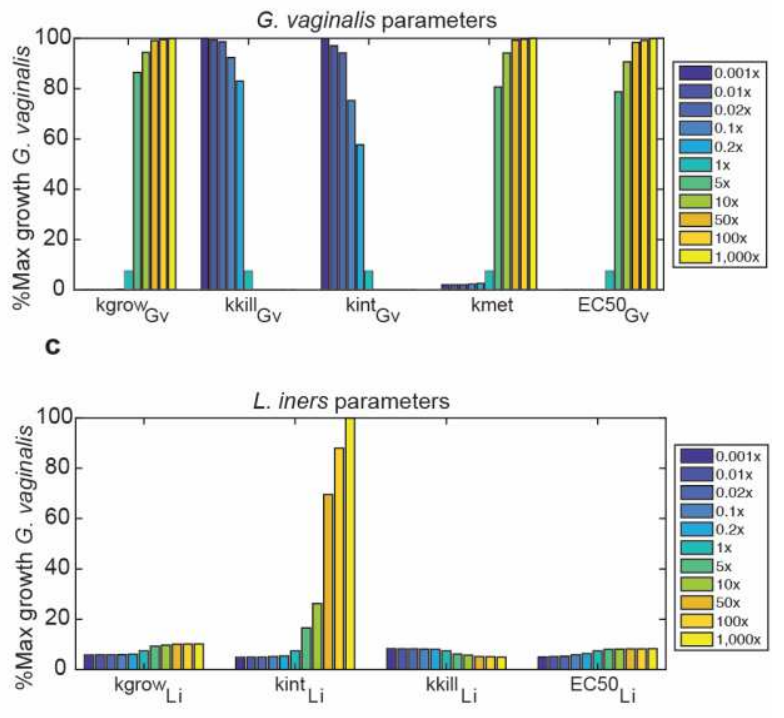

e

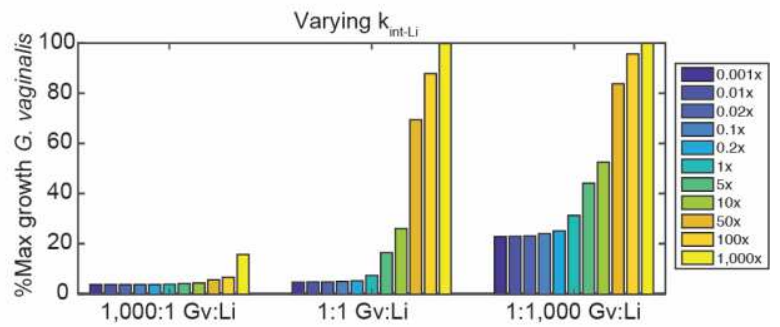

Figure 1: Model schematic for bacterial growth dynamics in BV with MNZ treatment.

(a) MNZ is internalized by both $G$. vaginalis $(G v)$ and $L$. iners $(L i)$ at rates kint-Gv and kintLI, cells are proliferating at kgrow-GV and kgrow-LI and MNZ inhibits growth by kkill-GV and kkillLI. For G. vaginalis, a potential mechanism of MNZ resistance is the bacterial-mediated interactions to the drug leading to the formation of metabolites (kmet). (b) Sensitivity of $G v$ growth with $500 \mu \mathrm{g} / \mathrm{ml}$ MNZ when parameters directly related to $G v$ growth are varied $0.001 x$ to $1,000 x$ baseline values. Percent maximal growth refers to the final cell count compared to the carrying capacity of the culture, or the maximum cell count the unperturbed culture can reach at $48 \mathrm{~h}$ based on initial cell counts (c) Sensitivity of $G v$ 
growth with $500 \mu \mathrm{g} / \mathrm{ml}$ MNZ when parameters related to $L i$ survival are varied $0.001 \mathrm{x}$ to 1,000x baseline values. (d) Max growth of $G v$ (left) and $L i$ (right) when the initial ratio of $G v$ to $L i$ is varied with $500 \mu \mathrm{g} / \mathrm{ml}$ MNZ treatment. (e) Max growth of $G v$ when MNZ internalization rate of $L i$ is varied at three different population compositions with $500 \mu \mathrm{g} / \mathrm{ml}$ MNZ treatment. 
a
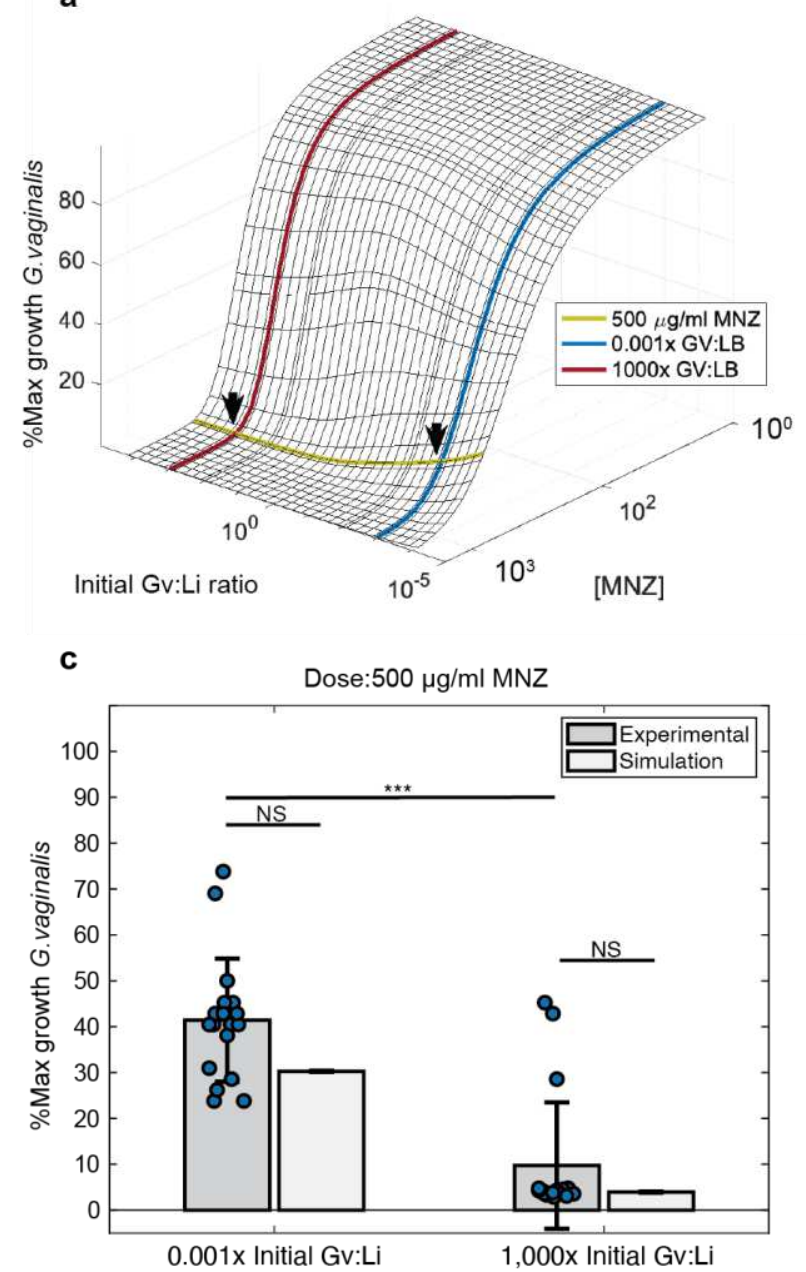

b
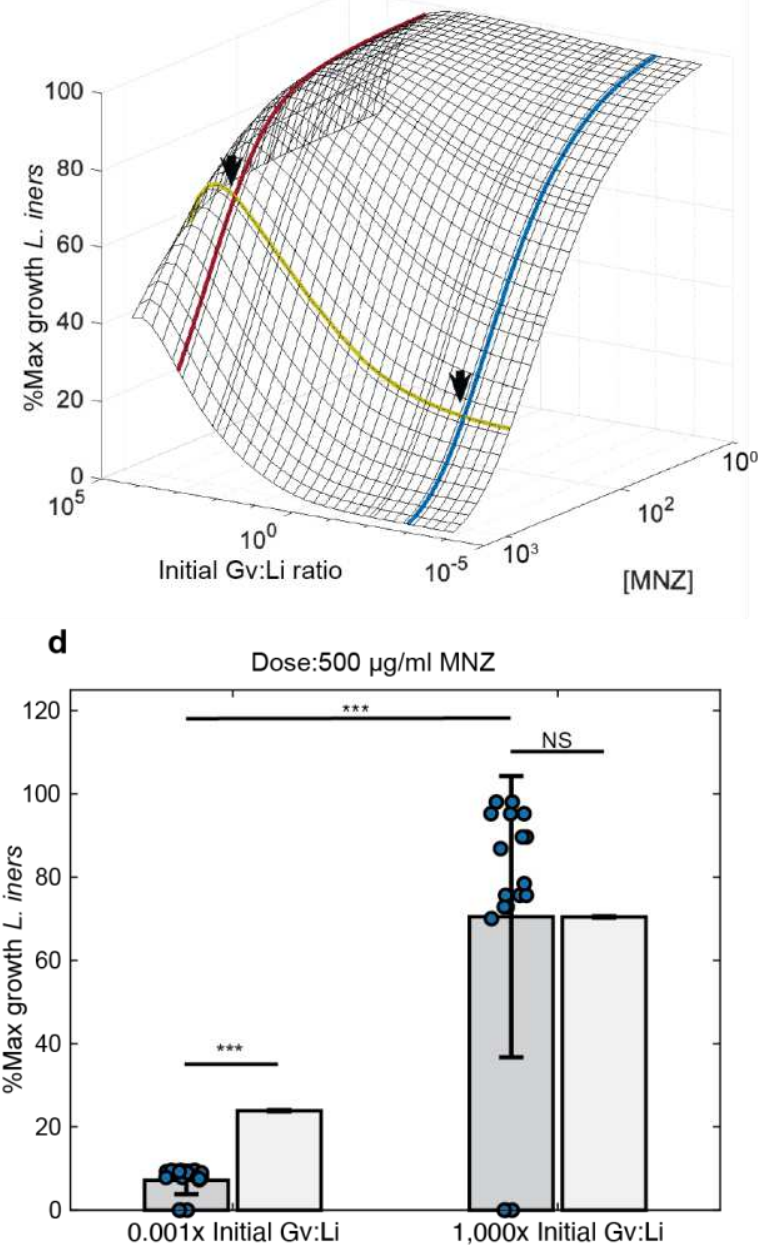

Figure 2: A higher initial Gv:Li ratio improves MNZ treatment efficacy. (a) Surface plot to illustrate predicted percent maximal growth of Gv (z-axis) when concentration of MNZ (x-axis) and the ratio of Gv:Li (y-axis) are varied in simultaneously. Arrows indicate the concentration of MNZ and ratios of Gv:Li used for model validation. (b) Percent maximal growth of $L i$ after simultaneous variation of MNZ dose and Gv:Li ratio. (c-d) Comparison of model simulations to experimental data for $500 \mu \mathrm{g} / \mathrm{ml} \mathrm{MNZ}$ at $1000 \mathrm{x}$ and $0.001 \times$ Gv:Li ( ${ }^{*} p<0.05,{ }^{* *} p<0.01,{ }^{* * *} p<0.001$, unpaired two-tailed t-test, error bars represent standard deviation). 


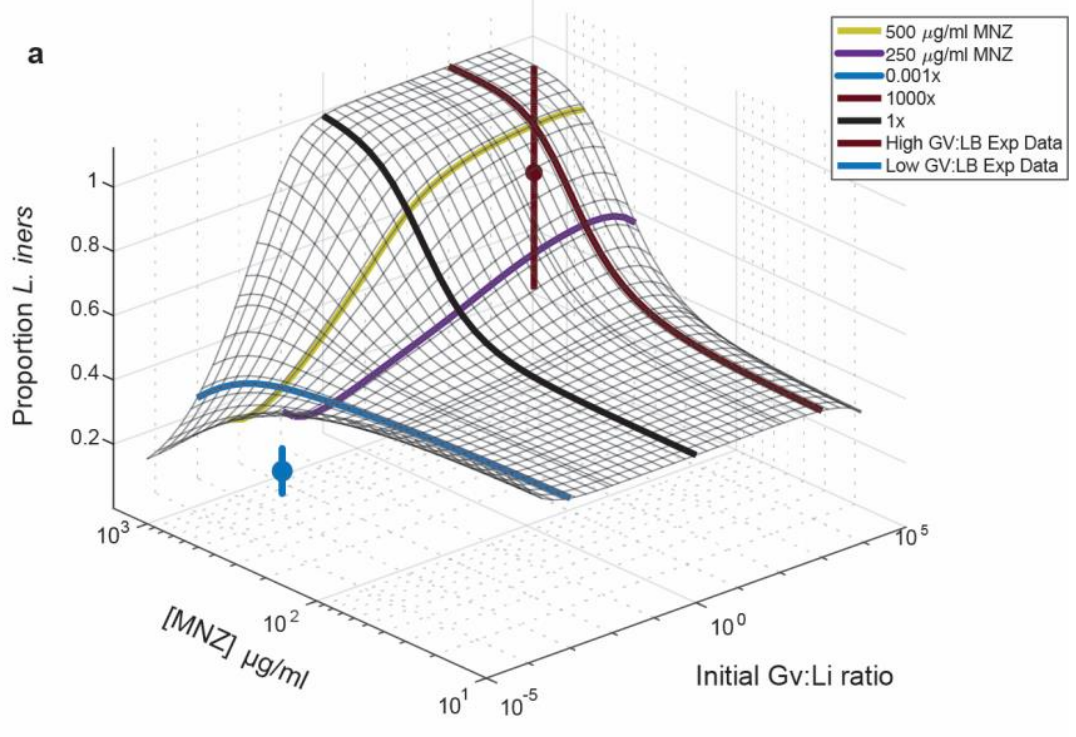

b

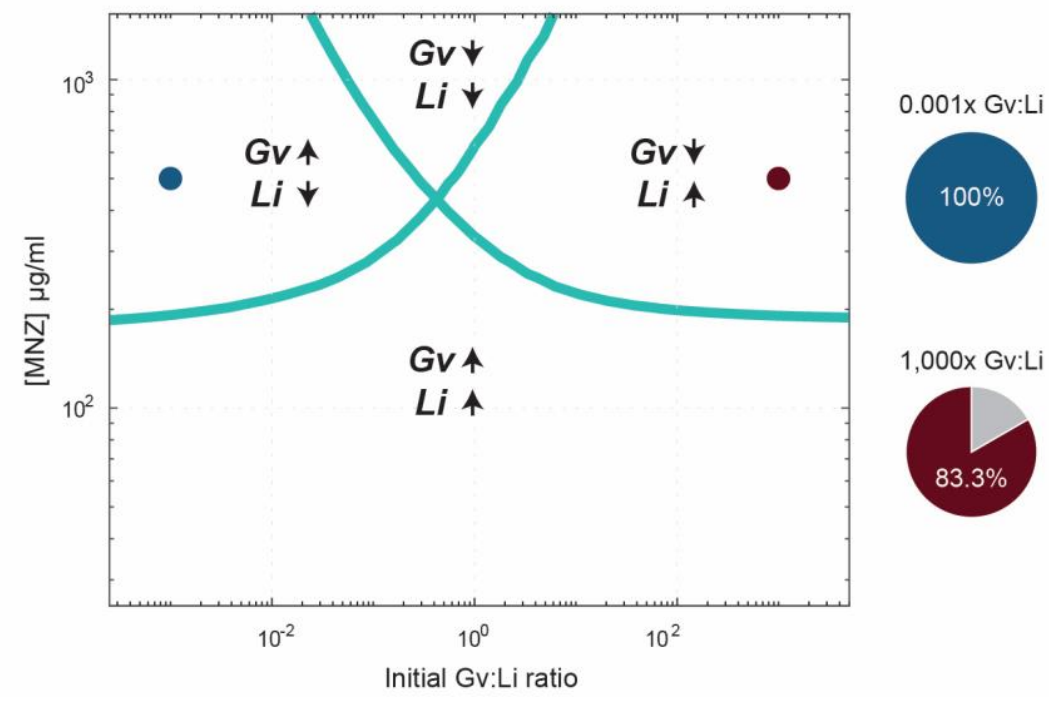

Figure 3: Initial Gv:Li ratios dictate final microbial populations. (a) Surface plot illustrates model predictions for proportion of $L i$ relative to $G v 48 \mathrm{~h}$ at different starting Gv:Li ratios (x-axis) and at different doses of MNZ (y-axis). Experimental validation was performed in in vitro co-cultures of $\mathrm{Li}$ and $\mathrm{Gv}(\mathrm{n}=36)$ and is plotted on the surface, with mean and \pm standard deviation represented by nodes and vertical lines (b) Phase diagram of microbial growth dynamics 48hrs after exposure to various MNZ doses, dots indicate experimental conditions evaluated. There are four possibilities: Both $G v$ and $L i$ 
populations are increased after treatment, both $G v$ and $L i$ populations are decreased, only the Gv population is increased and only the Li population is increased. Pie charts indicate the fraction of experimental samples that agree with the predicted trends (right). 
a

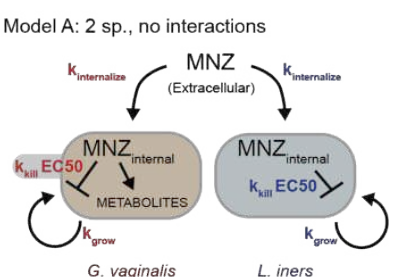

G. vaginalis

Model C: 4 sp., no interactions

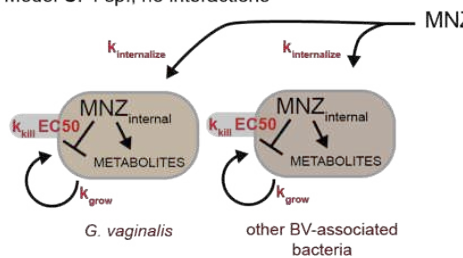

bacteria

Model D: 4 sp., with interactions

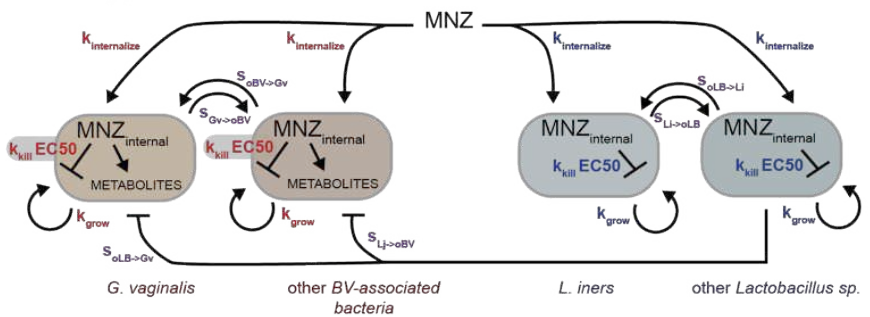

b Model B: 2 sp., with interactions

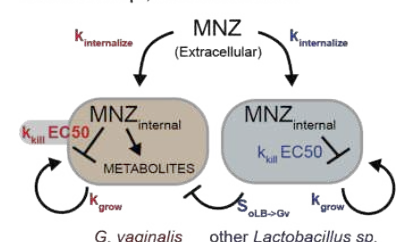

G. vaginalis other Lactobacillus sp.

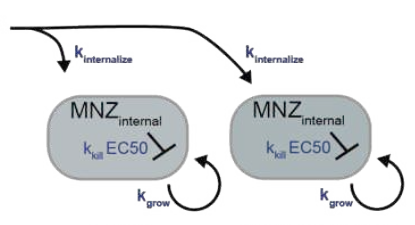

L. iners

other Lactobacillus sp.
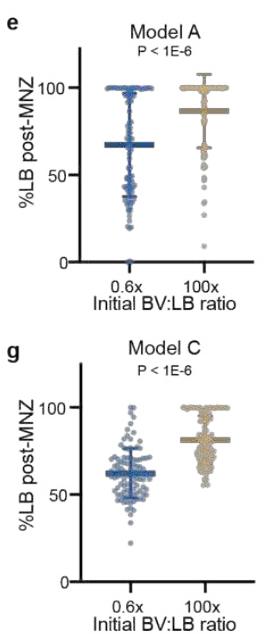
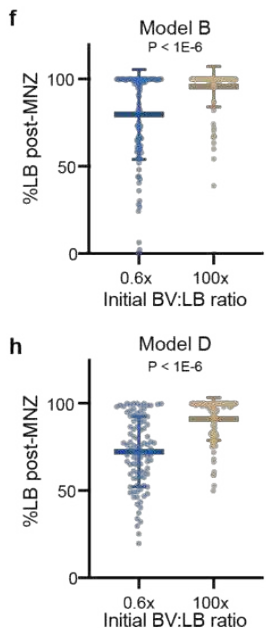

$$
\text { i }
$$

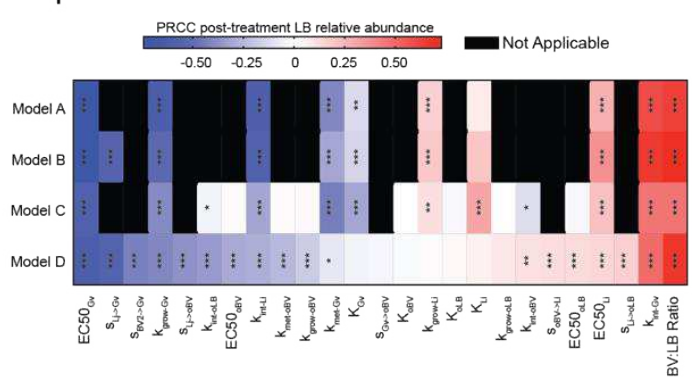

Figure 4: High pre-treatment BV:LB ratio is predicted to reduce MNZ efficacy in more complex microbial environments regardless of strain variability. (A) Original model structure validated in Fig. 2 (B) Two species model with negative interaction between other Lactobacillus sp. (oLB) and Gv (c) Four species model of Gv and Li with additional representative bacteria for BV-associated bacteria and Lactobacillus sp. (d) Four species model with inter-species interactions. Within BV-associated bacteria and Lactobacillus sp. interactions were simulated from mutualistic (both benefit) to commensal (one benefits, the other is neutral). Inhibitory (amensal) interactions are included between D-lactic acid producing bacteria, other Lactobacillus sp., with both BVassociated bacteria (e-h) post-MNZ treatment $(48 \mathrm{~h}, 500 \mu \mathrm{g} / \mathrm{ml})$ Lactobacillus sp. relative abundances at. $0.6 \mathrm{x}$ and 100x BV-associated bacteria to Lactobacillus sp (BV:LB) ratios. 
Each point represents a parameter set randomly sampled from physiological ranges in Tables S2 and S3 (i) Significantly sensitive parameters (assessed by partial rank correlation) for each model structure (a-d) as determined by the global sensitivity and uncertainty analysis. 

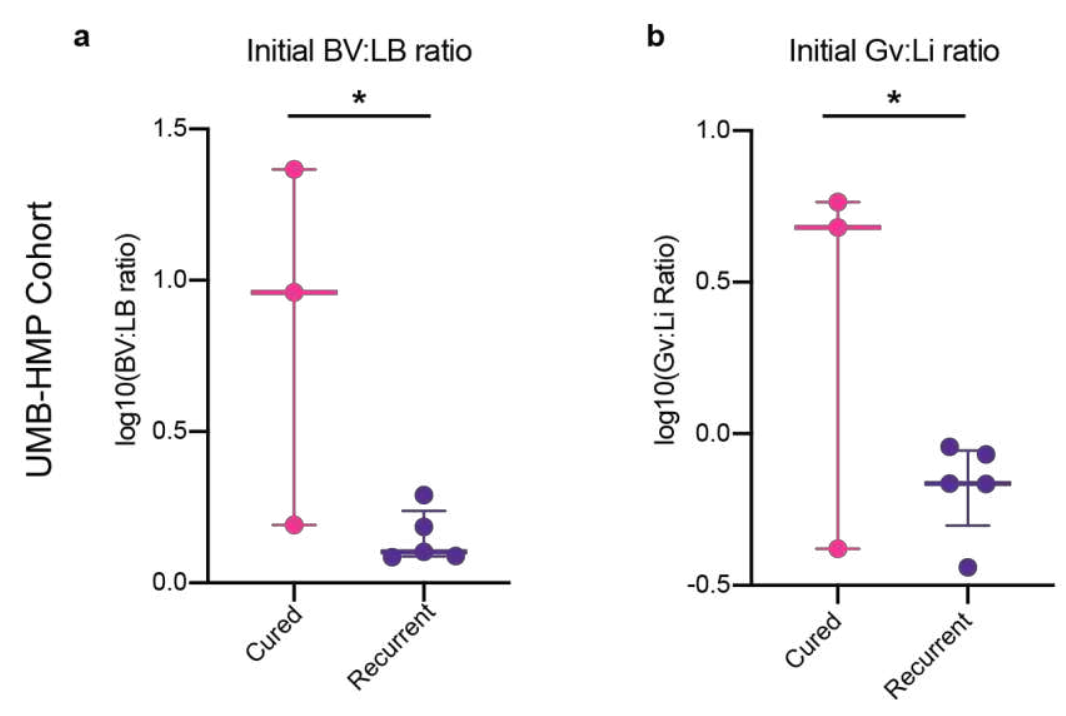

c

Initial BV:LB ratio

d
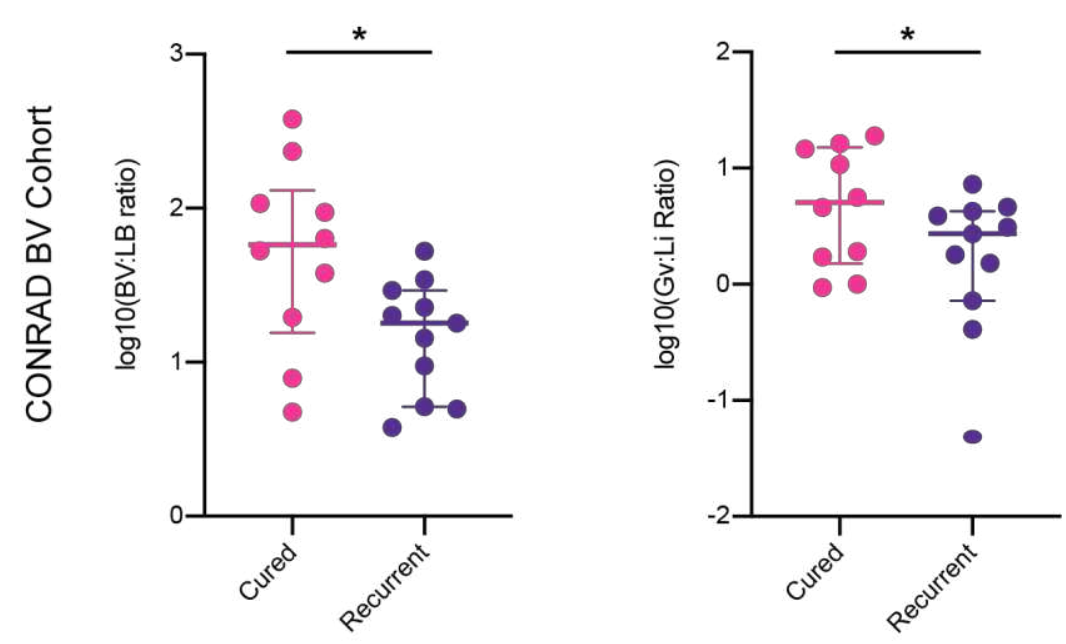

Figure 5: Increased initial BV:LB ratios associated with successful treatment of BV. $(a-b)$ clinical results for the UMB-HMP cohort describing the (a) log base 10 transform of initial BV-associated bacteria relative abundance to Lactobacillus sp. relative abundance (b) initial Gv:Li ratio. (c - d) Clinical results for the CONRAD BV cohort (c) log base 10 transform of initial BV-associated bacteria relative abundance to Lactobacillus sp. relative abundance. (d) initial Gv:Li ratio. Lines depict the 25th, 50th and 75th percentiles, multiple unpaired one-tailed t-test $p$-values were adjusted using Benjamini and Hochberg correction. 
a
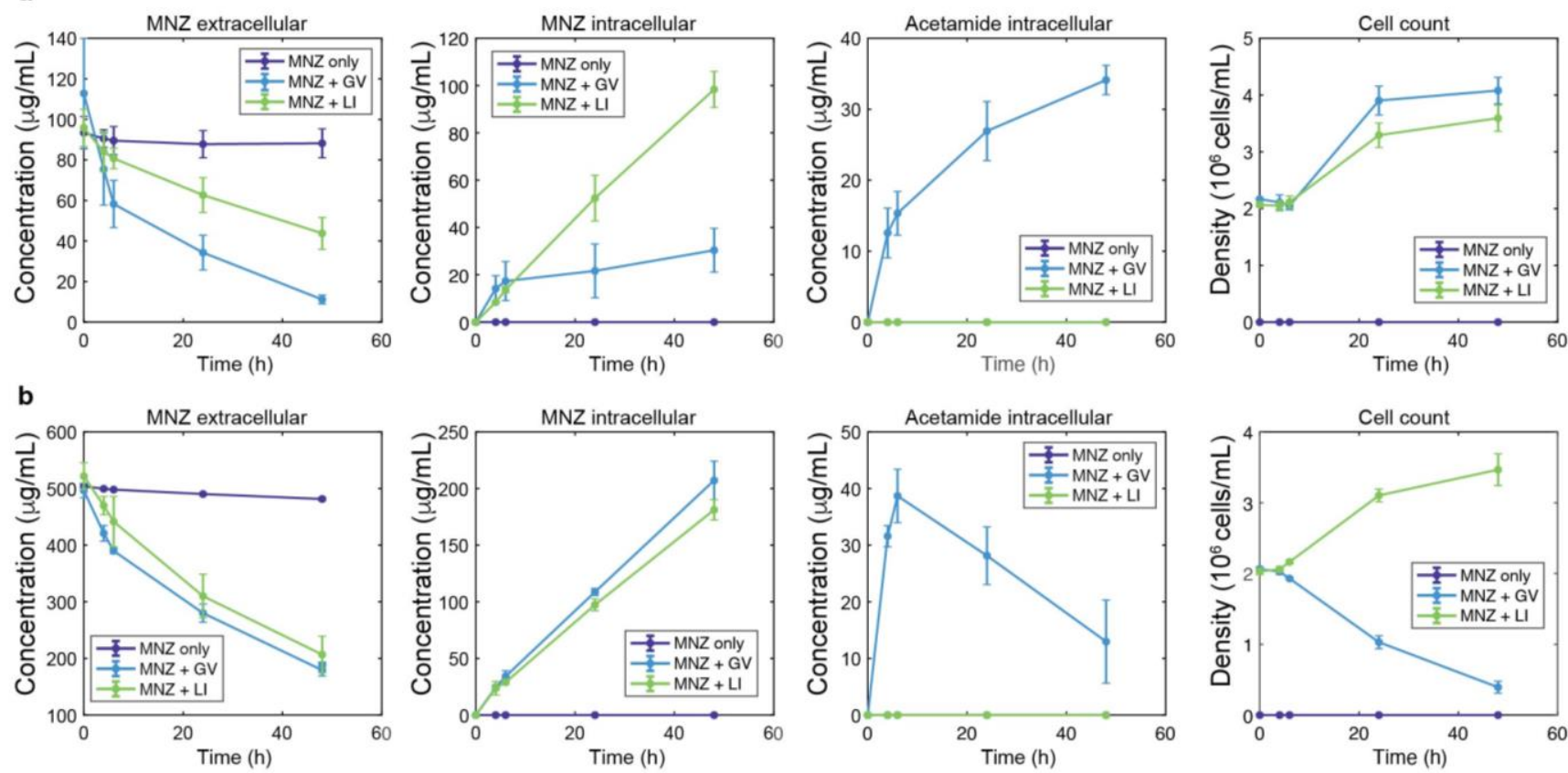

\section{Supplementary Figure 1: Kinetic data for parameterization of MNZ interactions with}

L. iners and G. vaginalis. Kinetic data was collected at two doses of MNZ, (a) low dose $(100 \mu \mathrm{g} / \mathrm{ml})$ and (b) high dose $(500 \mu \mathrm{g} / \mathrm{ml})$ for cultures with only MNZ, Gv and Li treated with MNZ (error bars $\pm S D$ ). 

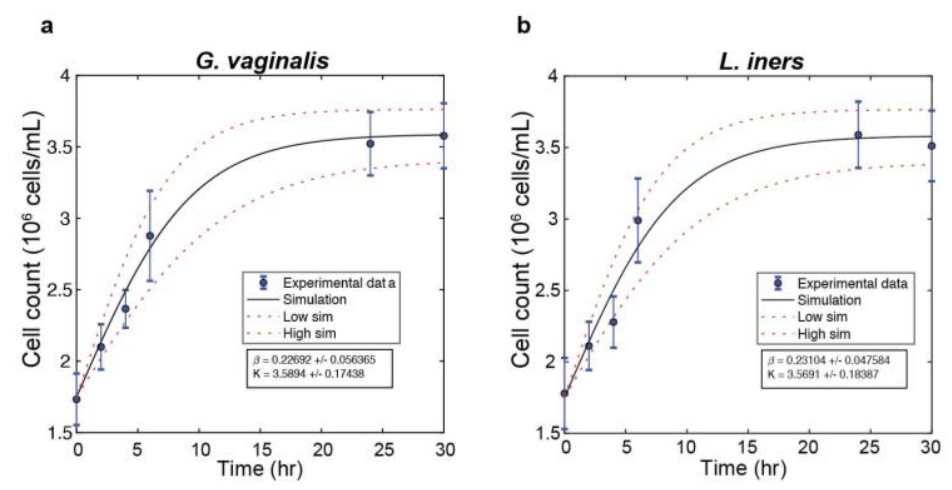

c

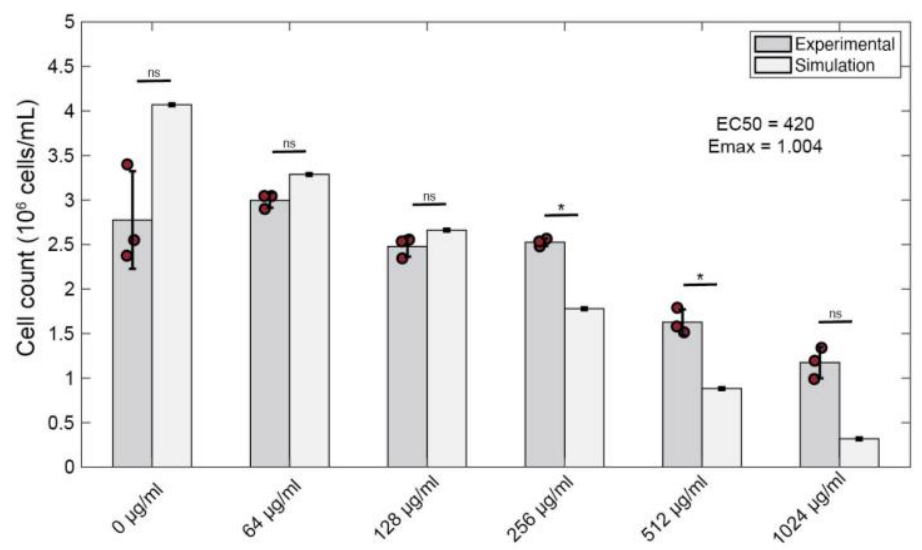

d

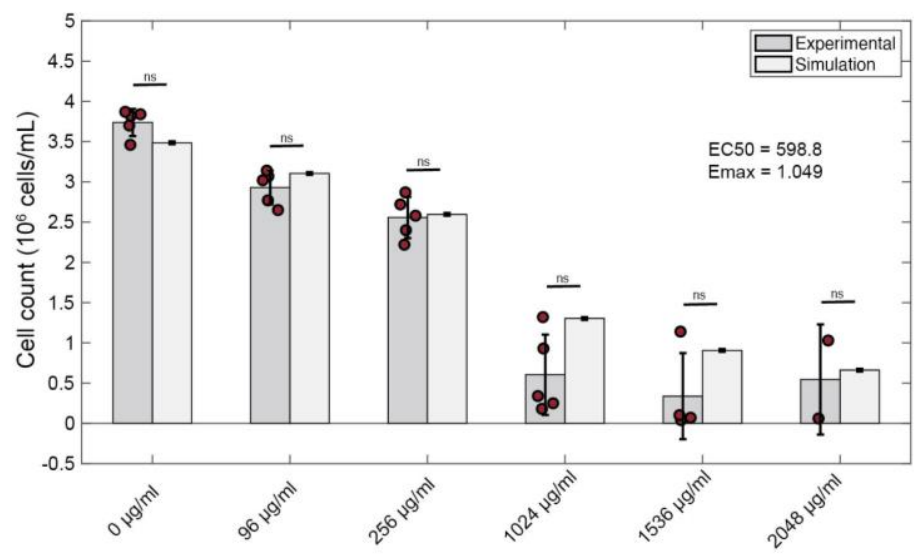

Supplementary Figure 2: Model parameterization of growth dynamics with and without MNZ. (a-b) growth curves fit to a logistic equation using least-squares regression, growth rates and carrying capacity was determined from this data. Red dashed lines represent confidence interval, values represent mean \pm SD (c-d) Kill curves used to determine the maximal kill rate and EC50 of MNZ for Gvand $L i$. 
a

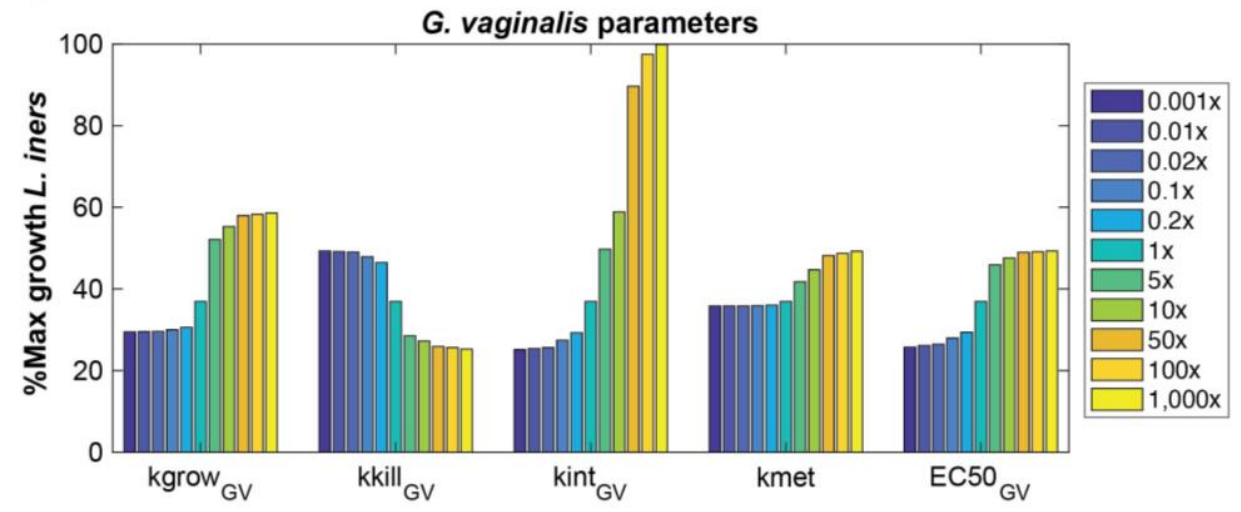

b

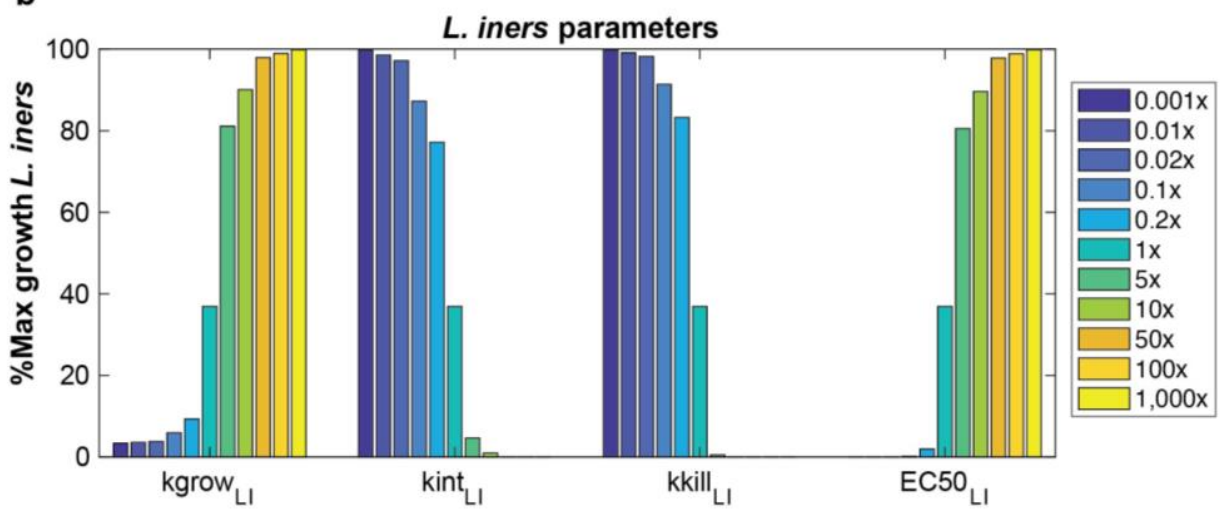

Supplementary Figure 3: L. iners 1D sensitivity analysis. (a) Sensitivity of Li growth with $500 \mu \mathrm{g} / \mathrm{ml}$ MNZ when parameters directly related to Ligrowth and survival are varied $0.001 x$ to $1,000 x$ fold baseline values. Percent maximal growth refers to the final cell count compared to the carrying capacity of the culture, the maximum cell count the culture can reach (b) Sensitivity of $L i$ growth with $500 \mu \mathrm{g} / \mathrm{ml}$ MNZ when parameters related to $L i$ survival are varied $0.001 \times$ to $1,000 x$ fold baseline values. 

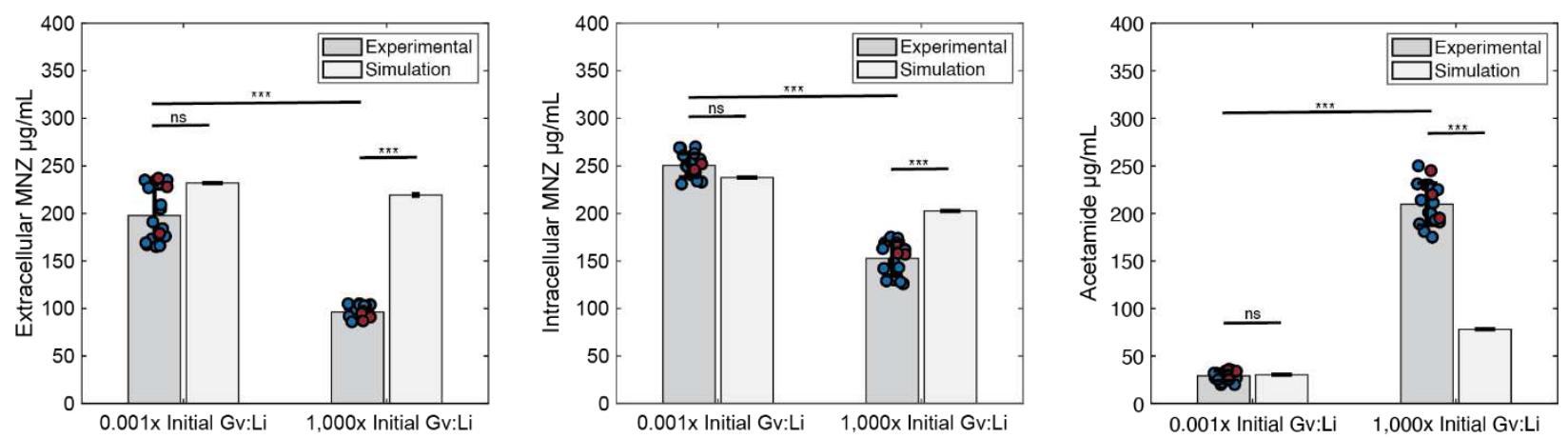

Supplementary Figure 4: Model validation. Validation for the model for extracellular MNZ, intracellular MNZ and acetamide. Intracellular MNZ is the sum of MNZ concentration in $L i$ and $G v$ in the model $\left({ }^{*} p<0.05,{ }^{* *} p<0.01,{ }^{* * *} p<0.001\right.$, unpaired two-tailed t-test, error bars represent standard deviation). 

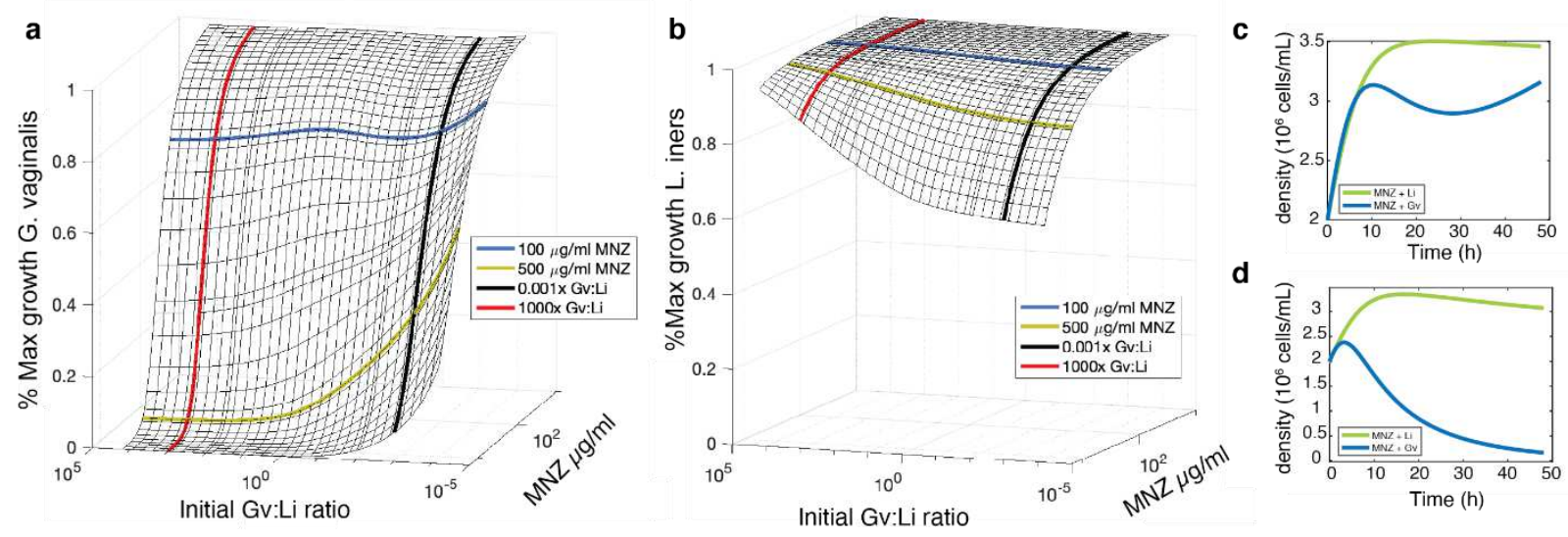

d

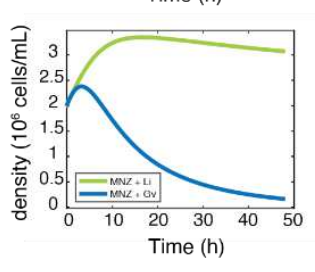

Supplementary Figure 5: $L$. iners susceptibility to MNZ does not influence Gv:Li ratio dependent MNZ efficacy. ( $a$ - b) Surface plot to illustrate predicted percent maximal growth of (a) Gvand (b) Li (z-axis) when concentration of MNZ (x-axis) and the initial ratio of Gv:Li (y-axis) are varied in simultaneously. $(c-d)$ Model predicted growth dynamics for monoculture response to MNZ at (c) $100 \mu \mathrm{g} / \mathrm{ml}$ and (d) $500 \mu \mathrm{g} / \mathrm{ml}$. 

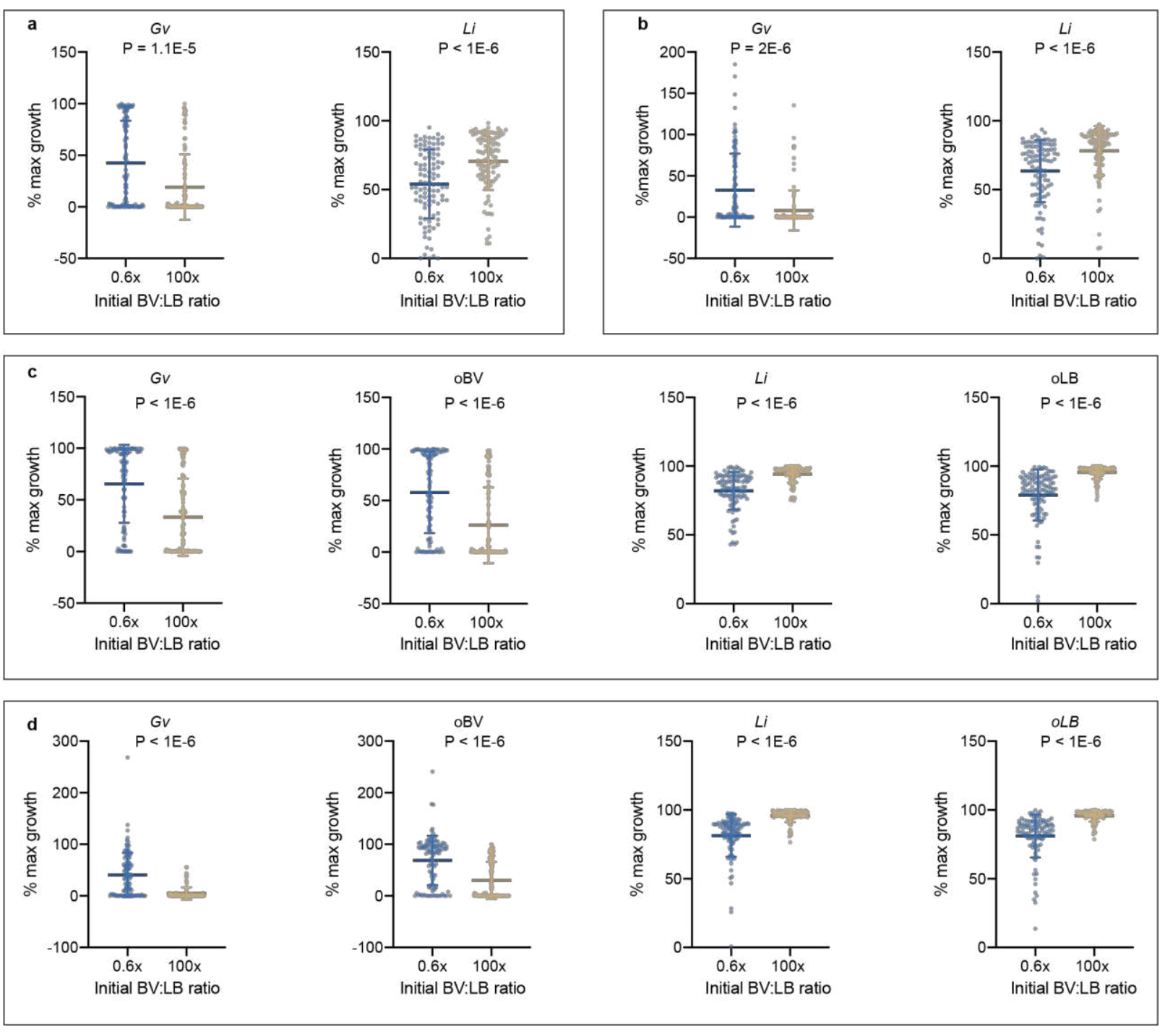

\section{Supplementary Figure 6: BV:LB ratios influence endpoint BV-associated bacteria} and Lactobacillus spp. abundances even with strain variation. (a) Model A: two species, no interactions proportion of maximal growth for Gv and Li. (b) Model B: two species with interactions proportion of maximal growth for Gv and Li. (c) Model C: four species, no interactions proportion of maximal growth for Gv, other BV-associated bacteria (oBV), $L i$, and other Lactobacillus sp. (oLB) (i - I) Model D: four species, with interactions proportion of maximal growth for Gv, other BV-associated bacteria (oBV), $L i$, and other Lactobacillus sp. (oLB). Lines indicate mean and standard deviation, 
multiple unpaired two-tailed t-test $p$-values were adjusted using Benjamini and Hochberg correction. 

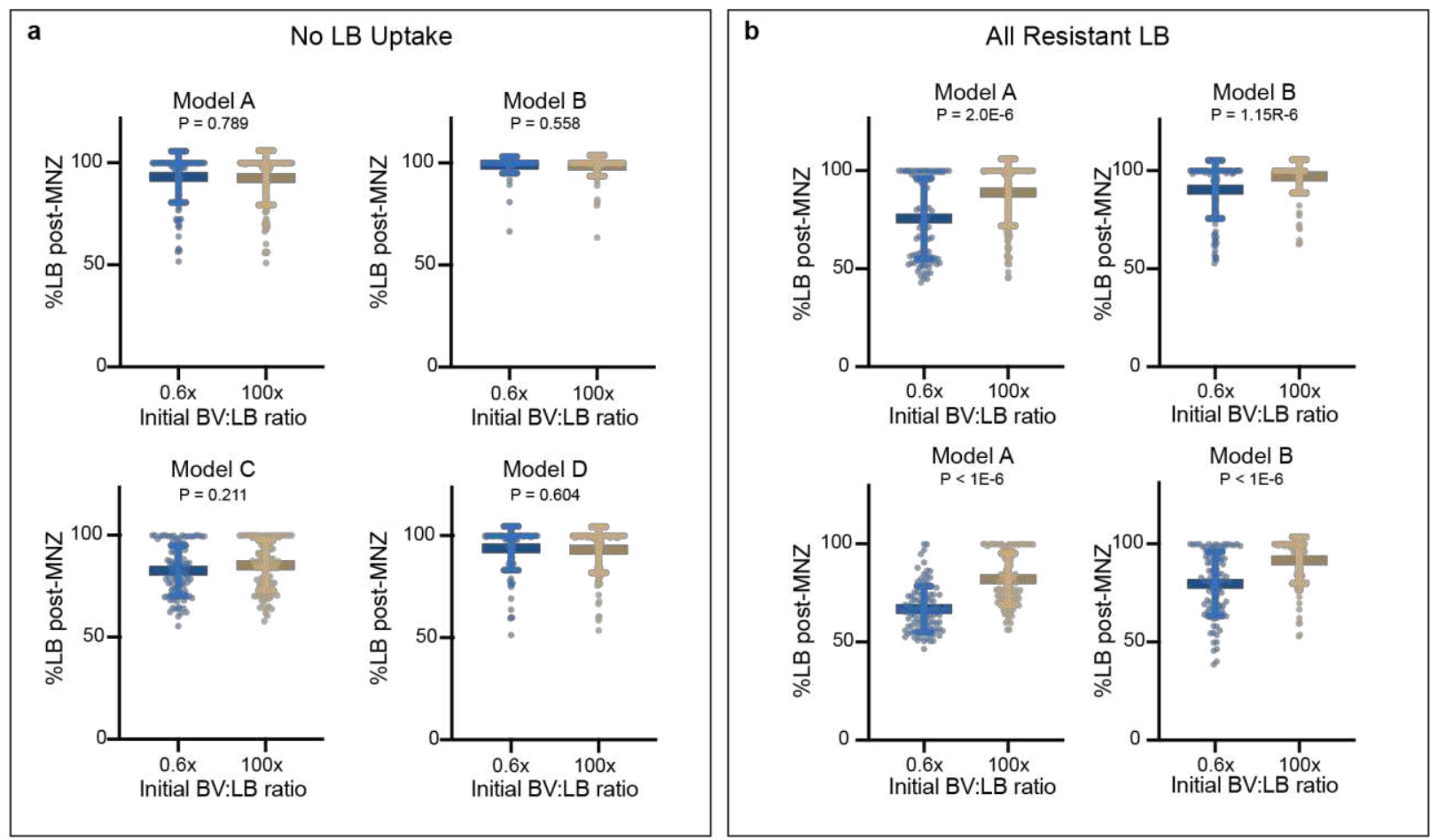

Supplementary Figure 7: Uptake or Sequestration of Lactobacillus spp. with MNZ drives initial BV:LB ratio influence of MNZ efficacy. (a) Post-treatment proportion of Lactobacillus sp. for Models A - D at $48 \mathrm{~h}$ with $500 \mu \mathrm{g} / \mathrm{ml} \mathrm{MNZ} \mathrm{with} \mathrm{the} \mathrm{rate} \mathrm{of}$ internalization of all Lactobacillus spp. (LB) set to zero (b) Post-treatment proportion of Lactobacillus sp. for Models A - D at $48 \mathrm{~h}$ with $500 \mu \mathrm{g} / \mathrm{ml} \mathrm{MNZ} \mathrm{with} \mathrm{the} \mathrm{EC50} \mathrm{set} \mathrm{to}$ 10,000 $\mu \mathrm{g} / \mathrm{ml}$ for all Lactobacillus spp. (LB). Lines indicate mean and standard deviation, multiple unpaired two-tailed t-test $p$-values were adjusted using Benjamini and Hochberg correction. 
a

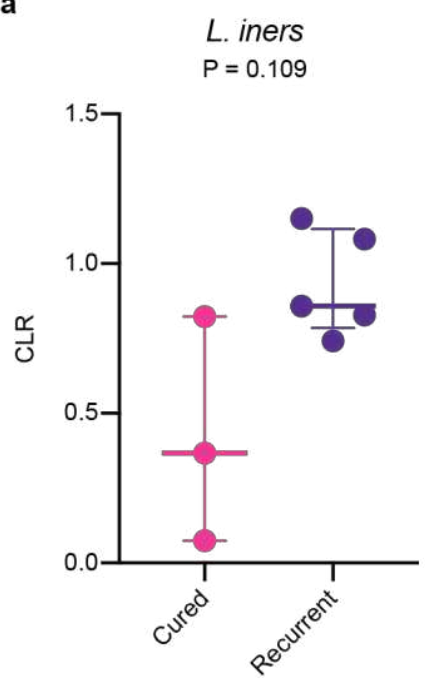

c

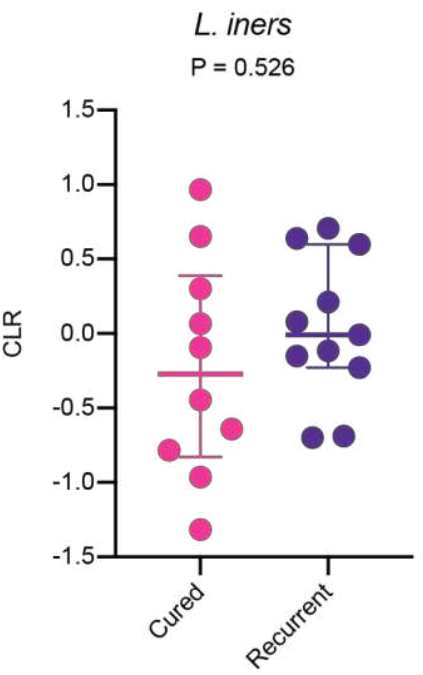

b

$$
\begin{gathered}
\text { G. vaginalis } \\
P=0.984
\end{gathered}
$$

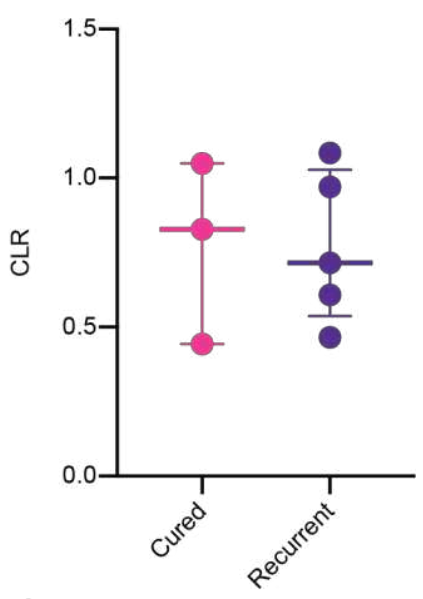

d

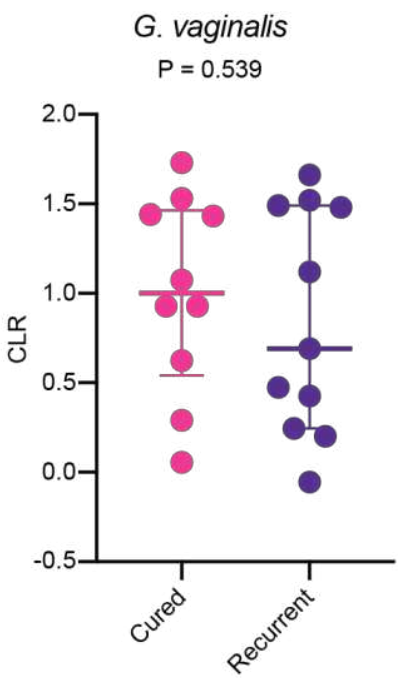

Supplementary Figure 8: Initial relative abundance data for $L$. iners and G. vaginalis for clinical outcomes. (a-b) UMB-HMP cohort for CLR-transformed relative abundances of (a) L. iners and (b) G. vaginalis (c-d) CONRAD BV cohort CLR-transformed relative abundances for (c) L. iners and (d) G. vaginalis. One-way unpaired t-test, central mark indicates median, bottom and top edges of the box represent the $25^{\text {th }}$ and $75^{\text {th }}$ percentiles and whiskers extend to most extreme data points that are not outliers. 


\section{Table S1: Model parameters.}

\begin{tabular}{|c|c|c|c|c|}
\hline Parameter Name & Description & Value & Units & Ref \\
\hline$k_{\text {int-GV }}$ & internalization rate of MNZ into GV & 0.0139 & cell density ${ }^{-1} \mathrm{hr}^{-1}$ & [1] \\
\hline kgrow-GV & maximal growth rate of GV & 0.2269 & $\mathrm{hr} \mathrm{r}^{-1}$ & [2] \\
\hline $\mathrm{K}_{\mathrm{GV}}$ & carrying a capacity for GV & 4.2 & $\begin{array}{l}\text { cell density } \\
\left(10^{6} / \mathrm{mL}\right)\end{array}$ & [2] \\
\hline$k_{\text {kill-GV }}$ & kill rate of MNZ on GV & 1.004 & $h r^{-1}$ & [3] \\
\hline $\mathrm{EC} 50_{\mathrm{GV}}$ & concentration of MNZ to kill $50 \%$ of GV & 420 & $\mu \mathrm{g} / \mathrm{mL}$ & [3] \\
\hline$k_{\text {met }}$ & $\begin{array}{l}\text { rate of MNZ conversion to unknown } \\
\text { metabolites }\end{array}$ & 0.0174 & cell density ${ }^{-1} \mathrm{hr}^{-1}$ & [1] \\
\hline$k_{\text {int }-L I}$ & internalization rate of MNZ into LI & 0.0042 & cell density ${ }^{-1} \mathrm{hr}^{-1}$ & [1] \\
\hline kgrow-LI & maximal growth rate of $\mathrm{LI}$ & 0.2309 & $h r^{-1}$ & [2] \\
\hline $\mathrm{K}_{\mathrm{LI}}$ & carrying a capacity for $\mathrm{LI}$ & 3.569 & $\begin{array}{l}\text { cell density } \\
\left(10^{6} / \mathrm{mL}\right)\end{array}$ & [2] \\
\hline k kill-Ll & kill rate of $\mathrm{MNZ}$ on $\mathrm{LI}$ & 1.049 & $h r^{-1}$ & [3] \\
\hline EC50 LI & concentration of MNZ to kill $50 \%$ of $\mathrm{LI}$ & 598.87 & $\mu \mathrm{g} / \mathrm{mL}$ & [3] \\
\hline
\end{tabular}

[1] Determined from fig. S1

[2] Determined from fig. S2a \& b

[3] Determined from fig. S2c \& d 
Table S2: Intraspecies Parameter Ranges

\begin{tabular}{|l|c|c|c|c|c|}
\hline & Units & G. vaginalis & $\begin{array}{c}\text { Other BV- } \\
\text { associated }\end{array}$ & L. iners & $\begin{array}{c}\text { Other } \\
\text { Lactobacillus }\end{array}$ \\
\hline 1) $\mathbf{k}_{\text {int }}$ & cell density ${ }^{-1} \mathrm{hr}^{-1}$ & $0.015-0.15$ & $0.0-0.20$ & $0.0015-0.15$ & $0.0-0.10$ \\
\hline 2) $\mathbf{k}_{\text {grow }}$ & $\mathrm{hr}^{-1}$ & $0.20-0.60$ & $0.20-0.40$ & $0.20-0.80$ & $0.20-1.00$ \\
\hline 3) $\mathbf{K}$ & $\begin{array}{c}\text { cell density } \\
\left(10^{6} / \mathrm{mL}\right)\end{array}$ & $3.0-4.5$ & $2.0-4.5$ & $3.0-5.0$ & $3.0-5.0$ \\
\hline 4) $\mathbf{E C 5 0}$ & $\mu \mathrm{g} / \mathrm{mL}$ & $50-500$ & $50-500$ & $400-4,000$ & $400-4,000$ \\
\hline 5) $\mathbf{K}_{\text {kill }}$ & $\mathrm{hr}^{-1}$ & 1 & 1 & 1 & 1 \\
\hline 6) $\mathbf{k}_{\text {met }}$ & cell density-1 & $0.005-0.05$ & $0.005-0.05$ & 0 & 0 \\
\hline
\end{tabular}

Table S3: Inter-species interaction terms. These terms descript the fold change in bacterial population that occurred from monoculture compared co-culture, a number greater than 1 indicates an increase in growth and less than 1 indicates an inhibition of growth.

\begin{tabular}{|c|c|c|c|c|c|}
\hline & \multicolumn{5}{|c|}{ Source $\left(f_{s->t}\right)$} \\
\hline \multirow{5}{*}{ Target } & & Gv & oBV & Li & OLB \\
\hline & Gv & & $1.0-1.3$ & $1.0-1.0$ & $1 \times 10^{-6}-1.0$ \\
\hline & OBV & $1.0-2.5$ & & $1.0-1.0$ & $1 \times 10^{-6}-1.0$ \\
\hline & Li & $1.0-1.0$ & $1.0-1.0$ & & $1.0-1.3$ \\
\hline & oLB & $1.0-1.0$ & $1.0-1.0$ & $1.0-1.3$ & \\
\hline
\end{tabular}


Table S4: Relative abundances of prevalent bacteria in BV for the UMB-HMP cohort.

\begin{tabular}{lllllll} 
Patient ID & L. iners & G. vaginalis & L. crispatus & A. vaginae & L. jensenii & other anaerobes \\
\hline UAB128 & 0.492 & 0.337 & 0.008 & 0.032 & 0.007 & 0.124 \\
UAB003 & 0.372 & 0.336 & 0.077 & 0.028 & 0.014 & 0.173 \\
UAB005 & 0.318 & 0.272 & 0.039 & 0.141 & 0.021 & 0.210 \\
UAB035 & 0.348 & 0.238 & 0.131 & 0.068 & 0.007 & 0.209 \\
UAB053 & 0.520 & 0.189 & 0.065 & 0.050 & 0.027 & 0.149 \\
UAB127 & 0.093 & 0.448 & 0.015 & 0.172 & 0.002 & 0.270 \\
UAB130 & 0.047 & 0.273 & 0.001 & 0.263 & 0.000 & 0.416 \\
UAB135 & 0.394 & 0.164 & 0.033 & 0.074 & 0.002 & 0.333 \\
\hline
\end{tabular}

Grey shading denotes patients that exhibited recurrent $B V$. 
Table S5: Relative abundances of prevalent bacteria in BV for the CONRAD BV cohort.

\begin{tabular}{llll} 
Patient ID & Gv:Li Ratio & G. vaginalis & L. iners \\
\hline 24_v1 & 4.606 & 0.348 & 0.075 \\
25_v1 & 1.793 & 0.030 & 0.017 \\
26_v1 & 0.051 & 0.008 & 0.161 \\
27_v1 & 4.257 & 0.017 & 0.004 \\
28_v1 & 1.519 & 0.063 & 0.042 \\
29_v1 & 3.882 & 0.021 & 0.006 \\
30_v1 & 0.722 & 0.015 & 0.020 \\
31_v1 & 3.096 & 0.125 & 0.040 \\
33_v1 & 7.276 & 0.145 & 0.020 \\
34_v1 & 0.408 & 0.054 & 0.133 \\
35_v1 & 2.726 & 0.345 & 0.126 \\
14_v1 & 5.592 & 0.121 & 0.022 \\
15_v1 & 4.602 & 0.032 & 0.007 \\
16_v1 & 18.962 & 0.067 & 0.004 \\
17_v1 & 14.635 & 0.124 & 0.009 \\
18_v1 & 1.916 & 0.182 & 0.095 \\
19_v1 & 0.933 & 0.016 & 0.018 \\
20_v1 & 1.000 & 0.015 & 0.015 \\
21_v1 & 16.337 & 0.034 & 0.002 \\
22_v1 & 1.723 & 0.224 & 0.130 \\
23_v1 & 10.784 & 0.013 & 0.001 \\
\hline
\end{tabular}

Grey shading denotes patients that exhibited recurrent BV. 


\section{Base Model Equations}

(1) $\frac{d[L i]}{d t}=\left[G_{L i}-D_{L i}\right] *[L i]$

(2) $\frac{d[G v]}{d t}=\left[G_{G v}-D_{G v}\right] *[G v]$

(3) $\frac{d\left[M N Z_{\text {ext }}\right]}{d t}=-k_{\text {int_LI }} *\left[M N Z_{\text {ext }}\right] *[L i]-k_{\text {int_GV }} *\left[M N Z_{\text {ext }}\right] *[G v]$

(4) $\frac{d\left[M N Z_{\text {int_LI }}\right]}{d t}=k_{\text {int } \_L I} *\left[M N Z_{\text {ext }}\right] *[L i]-D_{L i} *[L i] *\left[M N Z_{\text {int }}\right]$

(5) $\frac{d\left[M N Z_{\text {int_GV }}\right]}{d t}=k_{\text {int_GV }} *\left[M N Z_{\text {ext }}\right] *[G v]-k_{m e t} *\left[M N Z_{\text {int }}\right.$ GV $] *[G v]-D_{G v} *[G v] *\left[M N Z_{\frac{\text { int }}{G v}}\right]$

(6) $\frac{d[M e t]}{d t}=k_{m e t} *\left[M N Z_{\text {int }_{G V}}\right] *[G v]$

$D=k_{\text {kill }}\left(\frac{\left[M N Z_{\text {int }}\right]}{E C 50+\left[M N Z_{\text {int }}\right]}\right) \quad G=k_{\text {grow }} *\left(1-\frac{[\text { cell density }]}{K}\right)$

$M N Z_{\text {int } / \text { cell }}=\frac{\left[M N Z_{\text {int }}\right]}{[\text { cell density }] * V_{\text {culture }}} * V_{\text {cell }}$

Equation (1) represents the growth dynamics of the Li population, (2) the Gv population,

(3) the extracellular MNZ concentration (4) the bulk intracellular MNZ concentration for $L i$

(5) the bulk intracellular MNZ concentration for Gv (6) the concentration of metabolites

produced by Gv. "D" represents the death rate of the population and is dependent on the respective intracellular MNZ concentrations, EC50s and maximum kill rates. "G" represents the logistic growth of the respective populations dependent on the maximum growth rate and carrying capacities. "MNZ $Z_{\text {int/cell" }}$ represents the mass of MNZ internalized in each cell and is dependent on the bulk concentration of MNZ, per cell (cell density * culture volume) and cell volume. 


\section{Figures}

a

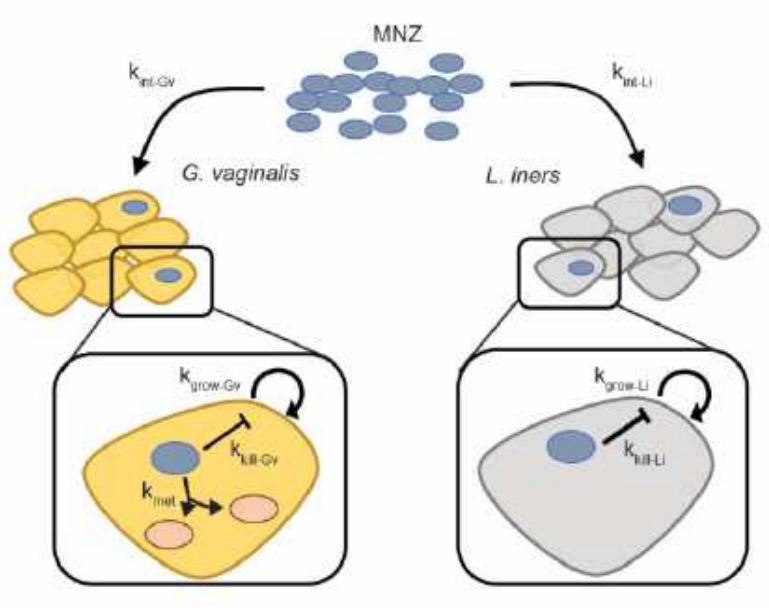

d

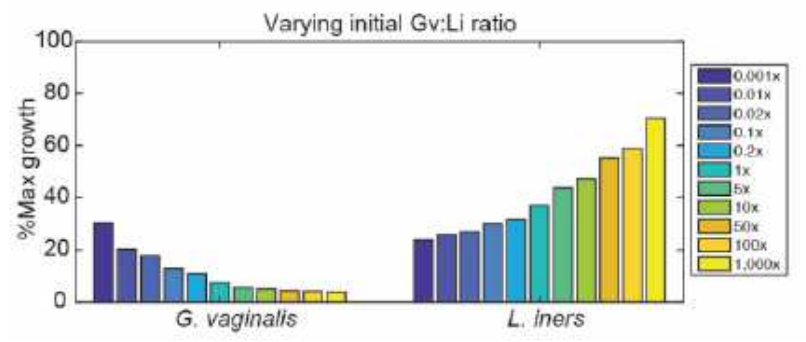

b
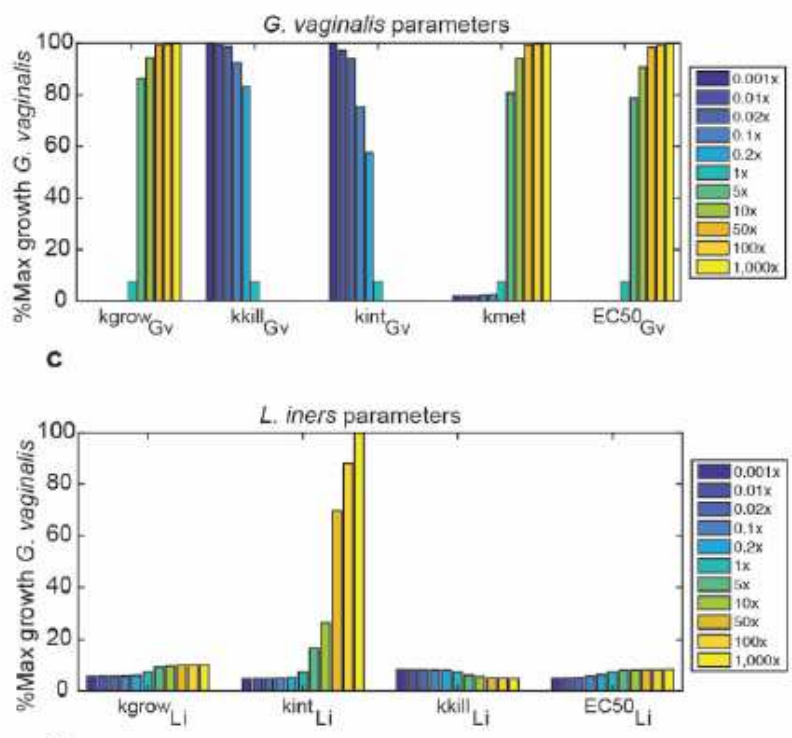

e

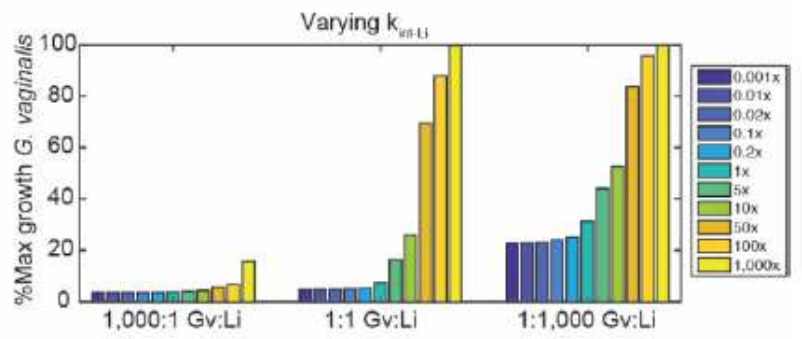

Figure 1

Model schematic for bacterial growth dynamics in BV with MNZ treatment. (a) MNZ is internalized by both $\mathrm{G}$. vaginalis $(\mathrm{Gv})$ and $\mathrm{L}$. iners $(\mathrm{Li})$ at rates kint-GV and kint-LI, cells are proliferating at kgrow-GV and kgrow-LI and MNZ inhibits growth by kkill-GV and kkill-LI. For G. vaginalis, a potential mechanism of MNZ resistance is the bacterial-mediated interactions to the drug leading to the formation of metabolites (kmet). (b) Sensitivity of Gv growth with $500 \mu \mathrm{g} / \mathrm{ml}$ MNZ when parameters directly related to Gv growth are varied $0.001 x$ to $1,000 x$ baseline values. Percent maximal growth refers to the final cell count compared to the carrying capacity of the culture, or the maximum cell count the unperturbed culture can reach at $48 \mathrm{~h}$ based on initial cell counts (c) Sensitivity of Gv growth with $500 \mu \mathrm{g} / \mathrm{ml} \mathrm{MNZ}$ when parameters related to Li survival are varied $0.001 x$ to $1,000 x$ baseline values. (d) Max growth of Gv (left) and $\mathrm{Li}$ (right) when the initial ratio of Gv to Li is varied with $500 \mu \mathrm{g} / \mathrm{ml}$ MNZ treatment. (e) Max growth of Gv when MNZ internalization rate of $\mathrm{Li}$ is varied at three different population compositions with 500 $\mu \mathrm{g} / \mathrm{ml} \mathrm{MNZ}$ treatment. 
a
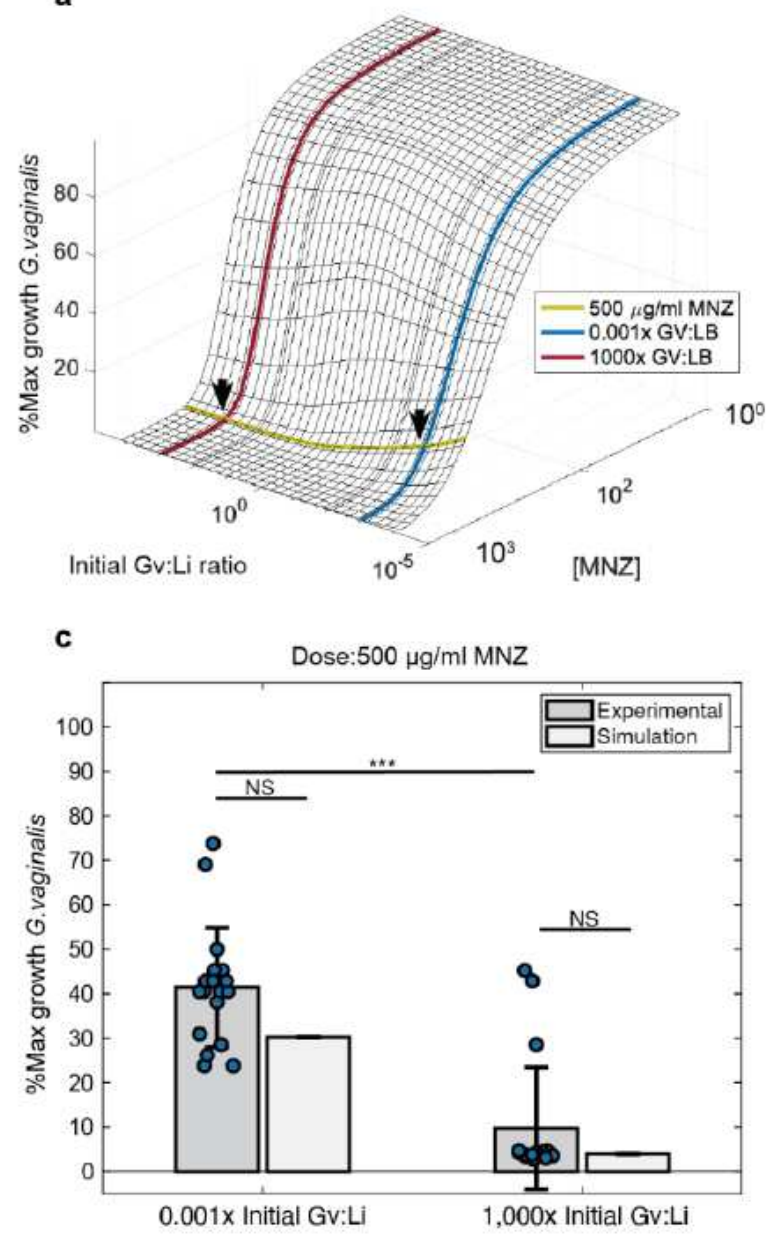

b

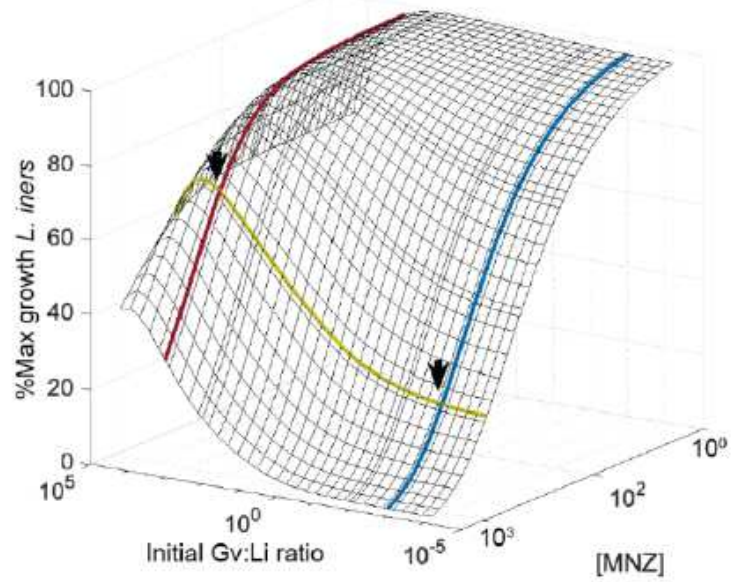

d

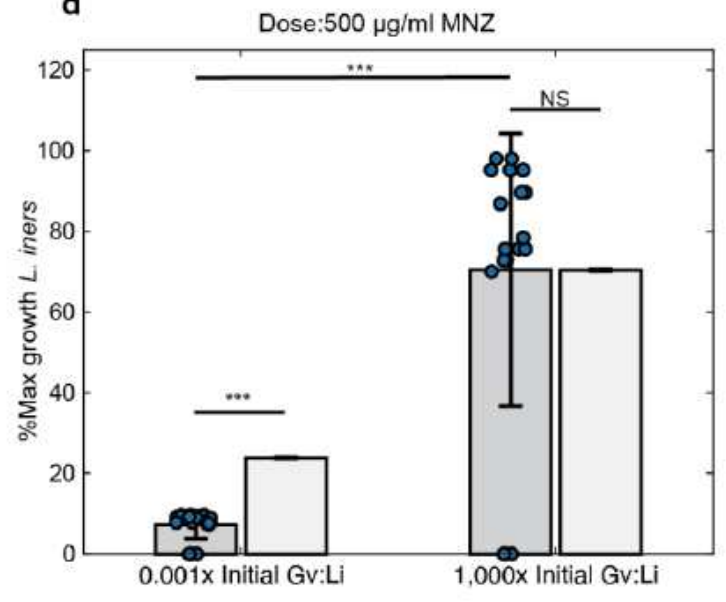

Figure 2

A higher initial Gv:Li ratio improves MNZ treatment efficacy. (a) Surface plot to illustrate predicted percent maximal growth of Gv (z-axis) when concentration of MNZ (x-axis) and the ratio of Gv:Li (y-axis) are varied in simultaneously. Arrows indicate the concentration of MNZ and ratios of Gv:Li used for model validation. (b) Percent maximal growth of Li after simultaneous variation of MNZ dose and Gv:Li ratio. (cd) Comparison of model simulations to experimental data for $500 \mu \mathrm{g} / \mathrm{ml} \mathrm{MNZ}$ at $1000 \mathrm{x}$ and $0.001 \mathrm{x} \mathrm{Gv}: \mathrm{Li}$ ( $\mathrm{p}<0.05,{ }^{\star *} \mathrm{p}<0.01,{ }^{\star \star *} \mathrm{p}<0.001$, unpaired two-tailed t-test, error bars represent standard deviation). 


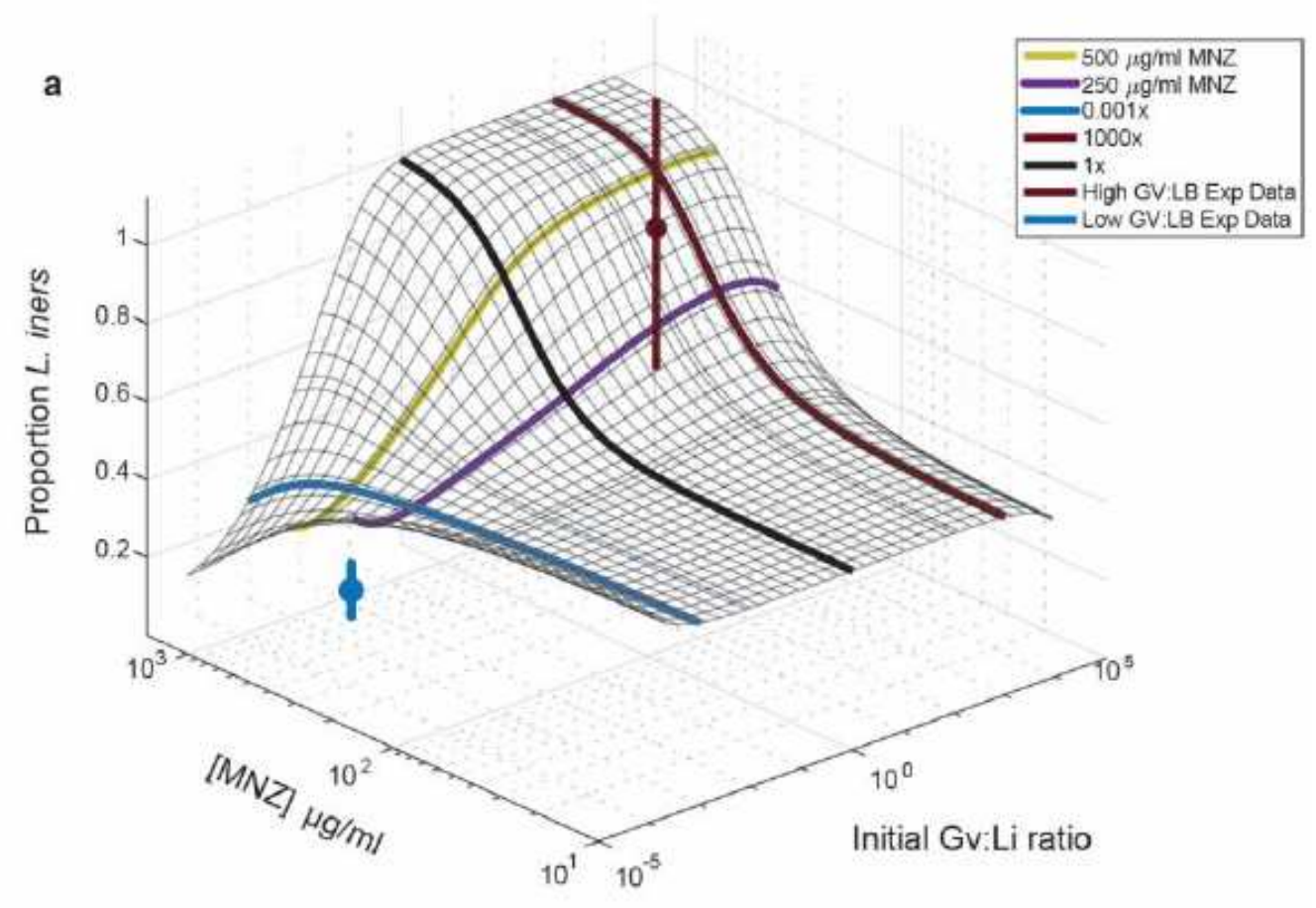

b

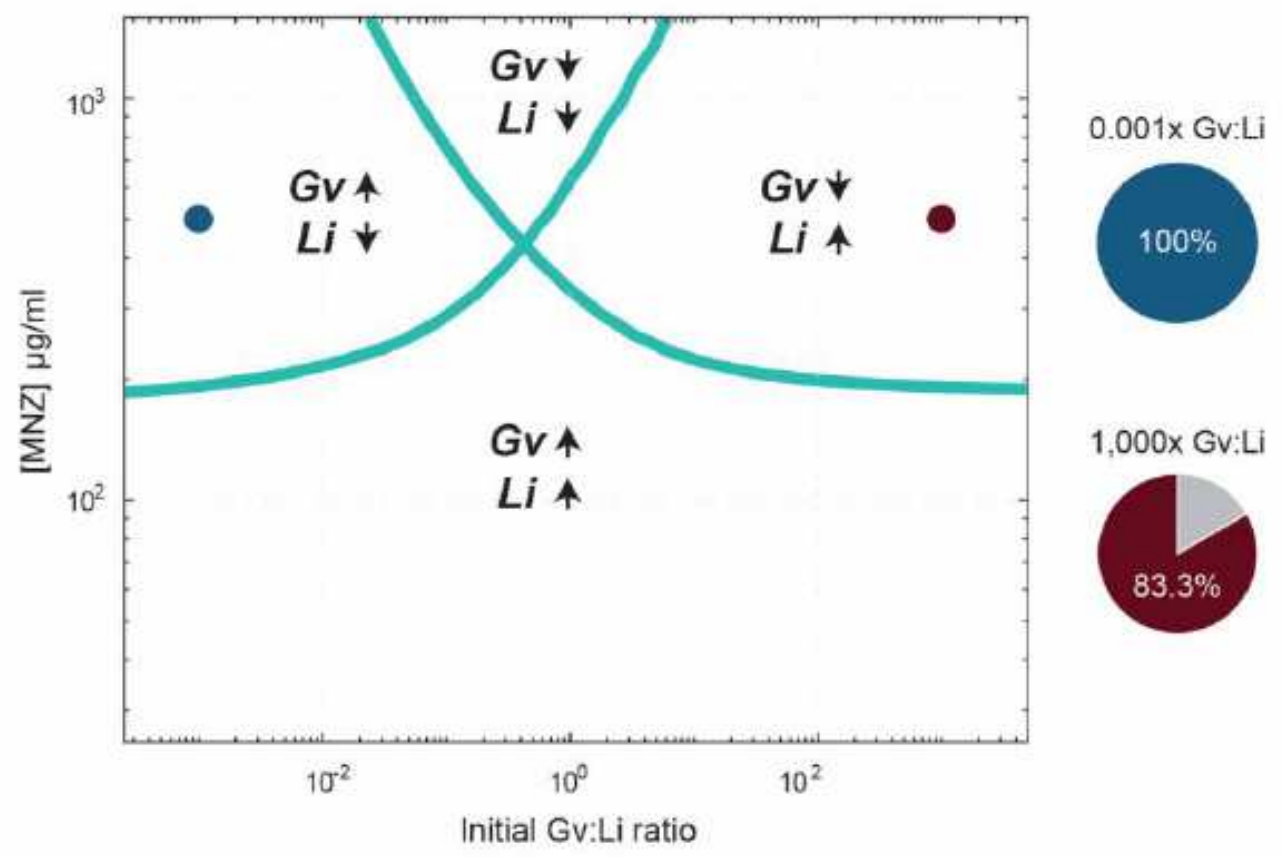

\section{Figure 3}

Initial Gv:Li ratios dictate final microbial populations. (a) Surface plot illustrates model predictions for proportion of Li relative to Gv 48h at different starting Gv:Li ratios (x-axis) and at different doses of MNZ (y-axis). Experimental validation was performed in in vitro co-cultures of $\mathrm{Li}$ and $\mathrm{Gv}(\mathrm{n}=36)$ and is plotted on the surface, with mean and \pm standard deviation represented by nodes and vertical lines (b) Phase diagram of microbial growth dynamics 48hrs after exposure to various MNZ doses, dots indicate 
experimental conditions evaluated. There are four possibilities: Both Gv and Li populations are increased after treatment, both Gv and Li populations are decreased, only the Gv population is increased and only the Li population is increased. Pie charts indicate the fraction of experimental samples that agree with the predicted trends (right).
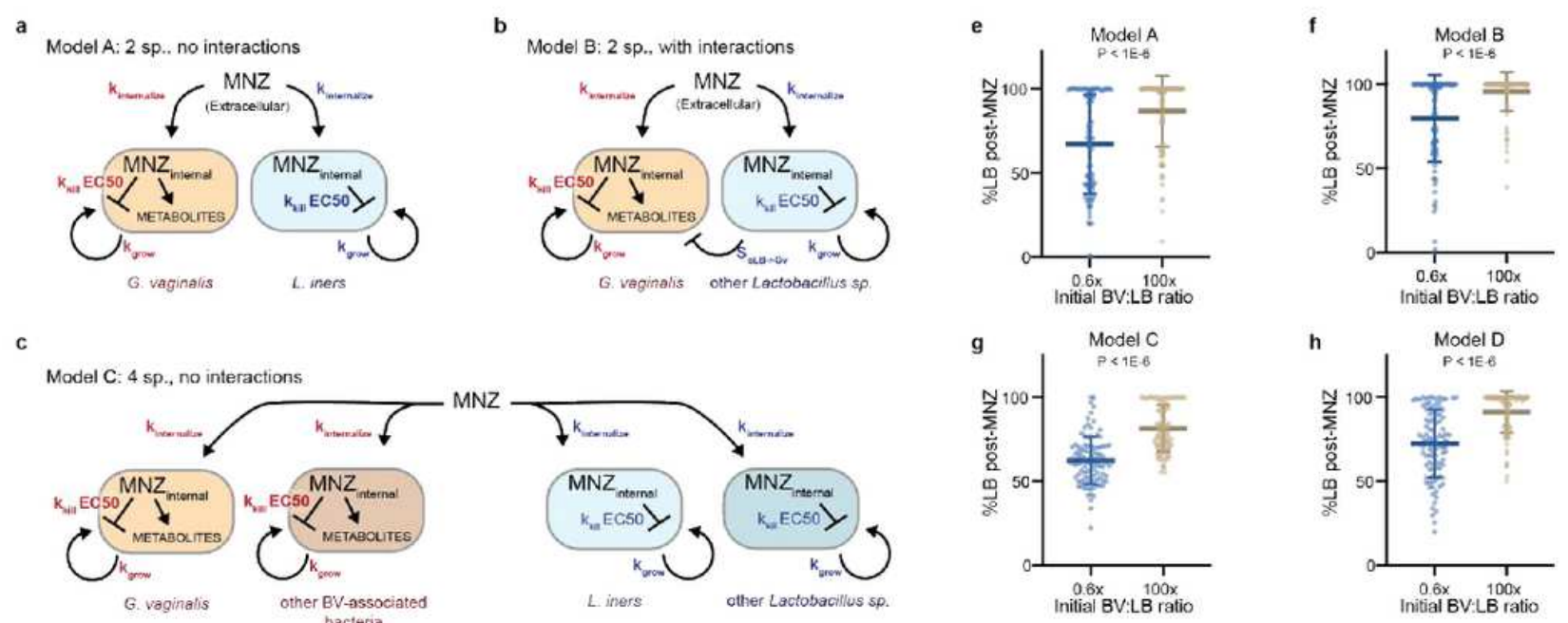

d
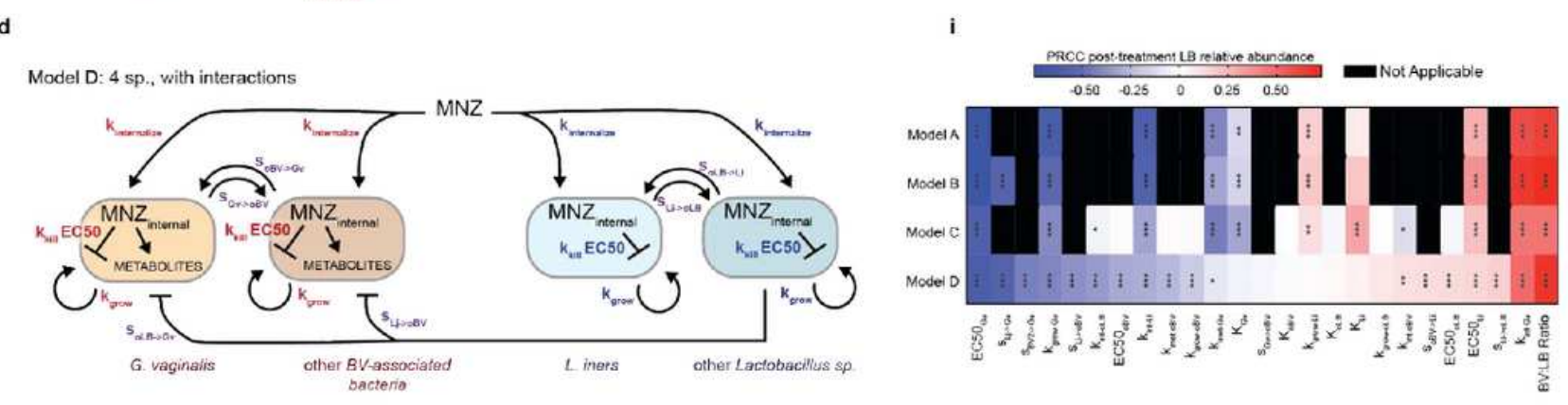

\section{Figure 4}

High pre-treatment BV:LB ratio is predicted to reduce MNZ efficacy in more complex microbial environments regardless of strain variability. (A) Original model structure validated in Fig. 2 (B) Two species model with negative interaction between other Lactobacillus sp. (oLB) and Gv (c) Four species model of Gv and Li with additional representative bacteria for BV-associated bacteria and Lactobacillus sp. (d) Four species model with inter-species interactions. Within BV-associated bacteria and Lactobacillus sp. interactions were simulated from mutualistic (both benefit) to commensal (one benefits, the other is neutral). Inhibitory (amensal) interactions are included between D-lactic acid producing bacteria, other Lactobacillus sp., with both BV-associated bacteria (e-h) post-MNZ treatment (48h, 500 $\mu \mathrm{g} / \mathrm{ml}$ ) Lactobacillus sp. relative abundances at. $0.6 \mathrm{x}$ and $100 \mathrm{x}$ BV-associated bacteria to Lactobacillus $\mathrm{sp}(\mathrm{BV}: \mathrm{LB})$ ratios. Each point represents a parameter set randomly sampled from physiological ranges in Tables S2 and S3 (i) Significantly sensitive parameters (assessed by partial rank correlation) for each model structure (a-d) as determined by the global sensitivity and uncertainty analysis. 
a

Initial BV:LB ratio

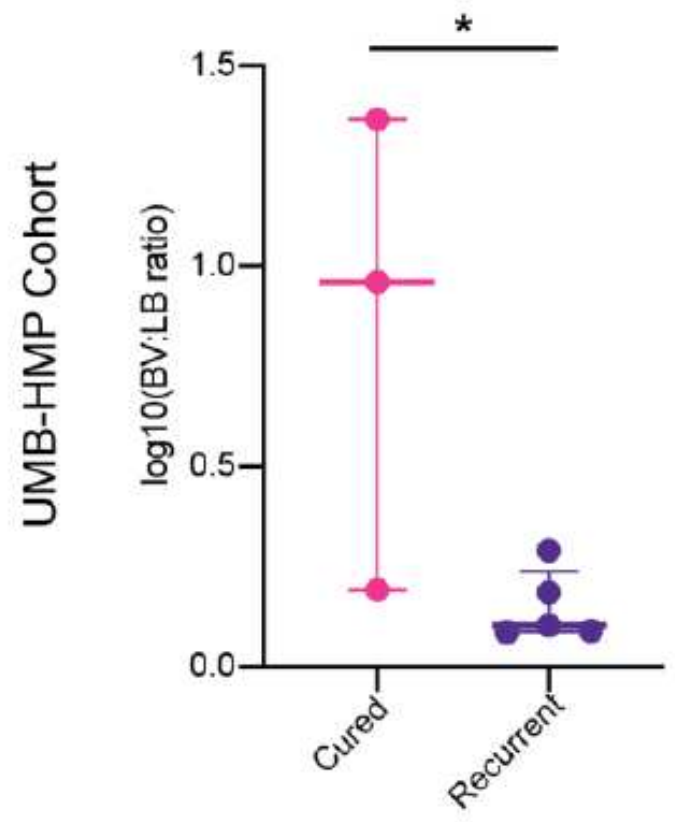

C

Initial BV:LB ratio

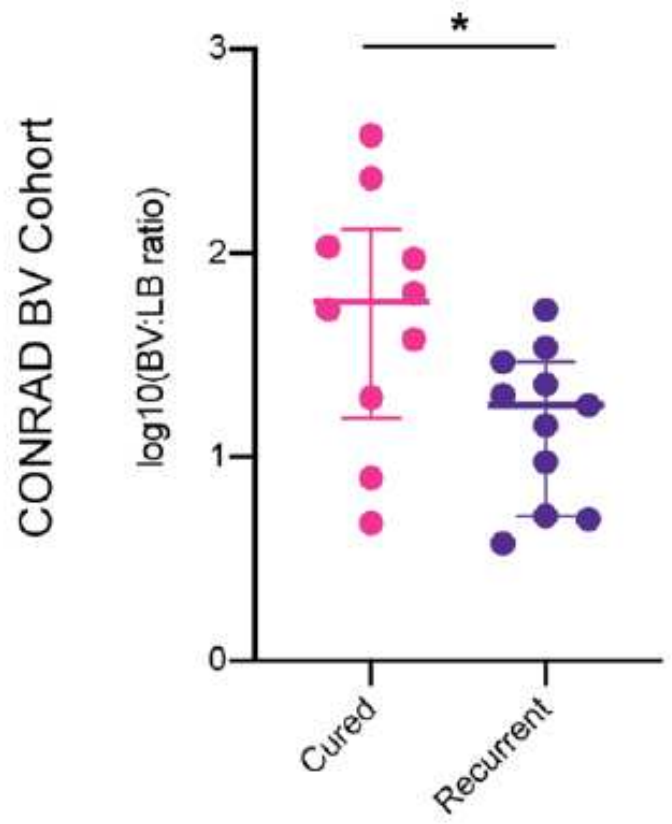

b

Initial Gv:Li ratio

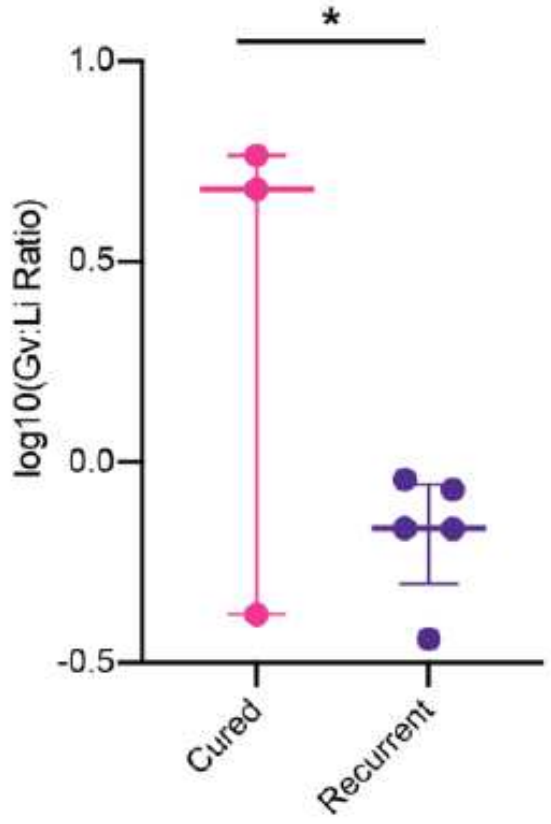

d

Initial Gv:Li Ratio

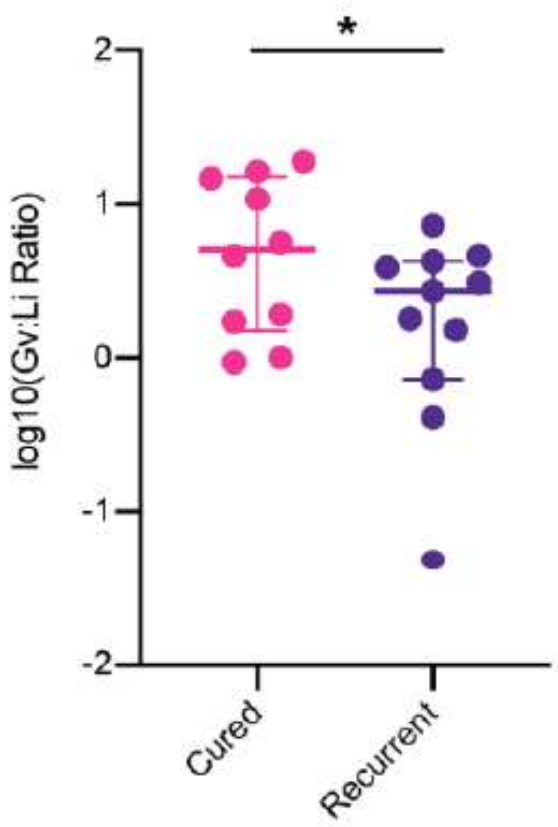

Figure 5

Increased initial BV:LB ratios associated with successful treatment of BV. $(a-b)$ clinical results for the UMB-HMP cohort describing the (a) log base 10 transform of initial BV-associated bacteria relative abundance to Lactobacillus sp. relative abundance (b) initial Gv:Li ratio. (c - d) Clinical results for the CONRAD BV cohort (c) log base 10 transform of initial BV-associated bacteria relative abundance to Lactobacillus sp. relative abundance. (d) initial Gv:Li ratio. Lines depict the 25th, 50th and 75th 
percentiles, multiple unpaired one-tailed t-test p-values were adjusted using Benjamini and Hochberg correction. 